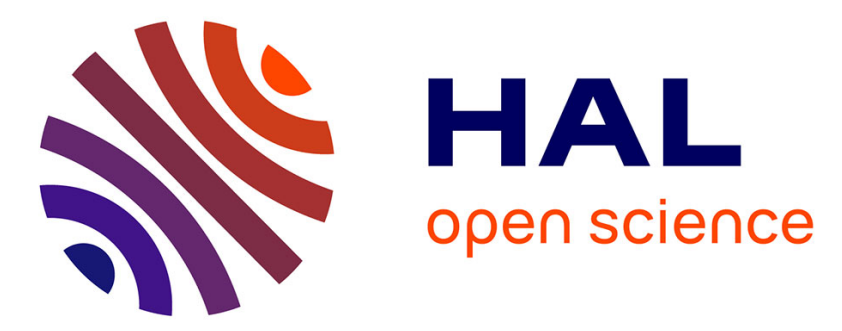

\title{
Les subordonnées complétives déclaratives et interrogatives indirectes dans un corpus italo-gaulois d'hagiographie médiolatine
}

Colette Bodelot

\section{- To cite this version:}

Colette Bodelot. Les subordonnées complétives déclaratives et interrogatives indirectes dans un corpus italo-gaulois d'hagiographie médiolatine. Anne Carlier et Céline Guillot-Barbance. Latin tardif, français ancien. Continuités et ruptures., De Gruyter, p. 323-372, 2018, 978-3-11-048963-7. hal01790051

\section{HAL Id: hal-01790051 \\ https://hal.science/hal-01790051}

Submitted on 9 Jun 2021

HAL is a multi-disciplinary open access archive for the deposit and dissemination of scientific research documents, whether they are published or not. The documents may come from teaching and research institutions in France or abroad, or from public or private research centers.
L'archive ouverte pluridisciplinaire $\mathbf{H A L}$, est destinée au dépôt et à la diffusion de documents scientifiques de niveau recherche, publiés ou non, émanant des établissements d'enseignement et de recherche français ou étrangers, des laboratoires publics ou privés. 
Colette BODELOT - Clermont Université, Laboratoire de Recherche sur le Langage

\section{Les subordonnées complétives déclaratives et interrogatives indirectes dans un corpus italo-gaulois d'hagiographie médiolatine*}

\section{Introduction}

L'expansion de la subordination explicite au détriment de la proposition infinitive à la suite d'un uerbum declarandi ou sentiendi passe pour un trait caractéristique de la syntaxe du latin tardif qui a été analysé dans différentes études portant sur des corpus variés ${ }^{1}$.

Dans une étude récente (Bodelot : 2014), portant sur la subordination complétive dans la Chronique originale de Frédégaire, un texte historique rédigé en latin mérovingien au $\mathrm{VII}^{\mathrm{e}} \mathrm{s}$. en Gaule, nous avons eu l'occasion de discuter et de nuancer l'hypothèse d'un emploi sans cesse croissant de la proposition conjonctionnelle (notamment en quod) aux dépens de l'A.c.I. (Accusatiuus cum Infinitiuo) en synchronie tardive. Le fait est que l'A.c.I. - qui y compte non moins de 130 occurrences - a étendu chez Frédégaire son domaine d'emploi après les verbes d'effort, d'ordre ou de permission et qu'après les uerba declarandi et sentiendi, quod p est avec 12 occurrences sûres - dont une seule à la suite d'un uerbum declarandi $(\mathrm{IV}, 71)$ - très peu représenté, un rival plus redoutable de l'A.c.I. que quod p étant $u t, \emptyset$, (ut) ne, (ut) non + subjonctif avec valeur déclarative.

Pour ce qui est des complétives interrogatives, nous avons pu constater que, dans la Chronique en question, le mode usuel dans l'interrogation indirecte est encore le subjonctif. Si l'indicatif y est employé, il n'admet guère de justification pragmatique ${ }^{2}$ ni ne peut être expliqué par l'absence d'un potentiel de doute ${ }^{3}$. Pour les termes interrogatifs, on observe, outre la confusion occasionnelle entre relatif et interrogatif ${ }^{4}$, que cur ne s'emploie chez Frédégaire que dans le sillage de verbes impliquant une interrogation associée à un état d'âme, ce qui a pu favoriser le passage de cur particule interrogative à $\mathrm{cur}$ conjonction causale ${ }^{5}$. Si, sporadiquement employé pour introduire une interrogation indirecte totale, y est toujours suivi du subjonctif.

Pour voir si les tendances observées chez Frédégaire se vérifient, à une échelle plus large, dans un corpus d'hagiographie italienne et gauloise s'échelonnant du $\mathrm{IV}^{\mathrm{e}}$ au XII ${ }^{\mathrm{e}} \mathrm{s} .{ }^{6}$, nous nous attacherons à analyser ici, plus particulièrement, les complétives assertives introduites par le verbe dico, à la suite duquel la concurrence entre propositions conjonctionnelles et A.c.I. est censée être devenue très âpre au fil des siècles ${ }^{7}$. Pour les interrogatives indirectes, nous nous limiterons à des observations ponctuelles concernant la syntaxe modale et certains termes interrogatifs, dont l'emploi particulier semble caractéristique de la latinité tardive et peut jeter de la lumière sur leur devenir ultérieur en roman.

\footnotetext{
* Je remercie vivement Sabine FIALON pour la relecture de la traduction des exemples cités dans cette étude.

${ }^{1}$ Pour un passage en revue rapide des principales études - notamment celles de J. Herman (1989), de M. Bolkestein (1989), de P. Cuzzolin (1994b), et, dans une plus large mesure, celle de L. Wirth-Pölchau (1977) - qui se sont intéressées à cette concurrence d'emplois, retracée occasionnellement jusqu'à Plaute, voir E. Karlsen (2001 : 16-24). Parmi les études publiées depuis le début de ce millénaire, contentons-nous de citer celles qui sont particulièrement développées : celle de G. Serbat (2003), qui est consacrée, dans une perspective à la fois diachronique et synchronique, à la description et à l'explication des emplois de quod complétif du latin préclassique au latin tardif, ainsi que celle, plus récente, de P. Greco (2012), qui traite de la subordination complétive dans les chroniques latines de l'Italie centro-méridionale du $\mathrm{X}^{\mathrm{e}}$ au XII ${ }^{\mathrm{e}} \mathrm{s}$.

${ }^{2}$ Voir à ce propos L. Stephens (1985 [1986]) ; C. Bodelot (1987 : $93-98$; 1999 ; 2003 : 297-314).

${ }^{3}$ Sur cette explication, voir D. Wanner $(1990: 266)$.

${ }^{4}$ Sur la fusion formelle entre pronoms relatifs et interrogatifs, probablement due à la proximité sentie entre les deux types de propositions que relatif et interrogatif sont censés introduire respectivement, voir V. Väänänen $\left(1981^{3}: 125\right)$.

5 Pour plus de détails sur cet emploi, voir J. Herman (1957 : 369-371) et J. B. Hofmann \& A. Szantyr (1972² : $541)$.

${ }^{6}$ Étude basée sur le corpus hagiographique médiolatin du LASLA; pour plus de détails, concernant la provenance, la date de rédaction (souvent incertaine) et la taille des textes considérés, voir l'introduction générale à ce volume (2.1.2. Description des textes) ainsi que le tableau reproduit à la fin de la présente étude. Pour d'autres études récentes sur corpus hagiographique, voir par ex. C. Philippart de Foy $(2008 ; 2015)$, S. Fialon (2012; à paraître).

${ }^{7}$ Voir par ex., dans le sillage de dico, la répartition de l'A.c.I. et de quod p dans les Revelaciones de sainte Brigitte de Suède au XIV ${ }^{\mathrm{e}}$ s. (Karlsen 2001 : 66-67).
} 


\section{Les déclaratives indirectes}

\subsection{Les propositions conjonctionnelles}

\subsection{1. quod p}

Quod introduit dans le sillage de dico beaucoup moins souvent une complétive déclarative que quia (5 occurrences de quod contre 12 de quia possibles ${ }^{8}$ ).

Tableau 1 : Types de complétives

\begin{tabular}{|l|l|l|l|l|}
\hline & A.c.I. & quia $p$ & quod $p$ & ut p \\
\hline antéposé & $17(27 \%)$ & $1(8,3 \%)$ & $0(0 \%)$ & $0(0 \%)$ \\
\hline postposé & $46(73 \%)$ & $11(91,7 \%)$ & $5(100 \%)$ & $1(100 \%)$ \\
\hline Total & $63(100 \%)$ & $12(100 \%)$ & $5(100 \%)$ & $1(100 \%)$ \\
\hline
\end{tabular}

Quatre des cinq occurrences de quod ont été repérées dans des textes rédigés en Italie à partir du $\mathrm{VI}^{\mathrm{e}}$ s., l'unique exemple attesté en Gaule est beaucoup plus tardif : il se rencontre chez Raoul de Cluny dans la Vita Petri Venerabilis, et ne date que du XII ${ }^{\mathrm{e}}$ s. ${ }^{9}$. Loin d'être libre, l'emploi de quod dans ce contexte semble être fortement conditionné. Pour ce qui est de l'ordre des mots, quod p est toujours postposé au verbe introducteur dico. Dans 4 cas sur 5, quod p a une structure complexe dans la mesure où une autre proposition subordonnée y est imbriquée :

(1) Grégoire, Vita Benedicti 2,16,57 (=82): Ad utraque haec tibi superius sub breuitate respondi dicens quod sancti uiri in quantum cum domino sunt sensum domini non ignorant. « Encontre cez dous choses ai a toi ci dessoure desoz brieteit respondut disanz, ke li saint homme en combien il un sont auoc lo sanior, si seuent il lo sens del sanior. ${ }^{10} /$ « Sur ces deux points, je t'ai répondu plus haut en quelques mots disant que les saints, dans la mesure où ils sont en communion avec le Seigneur, n'ignorent pas les pensées du Seigneur. »

(2) Passio Viti 1,11,6 (BHL 8712T): At illi cum eum penes supra memoratum fluuium orantem repperissent dixerunt quod imperator illum ad se accersiri propter quandam necessitatem iussisset.

«Mais comme ils l'avaient trouvé en train de prier près du fleuve mentionné plus haut, ils lui dirent que l'empereur avait ordonné de le faire venir chez lui pour un besoin impérieux. »

ou y est rattachée en remorque :

(3) Passio Caeciliae 1,26,25: Dicit eis Caecilia ite ergo et dicite infelici Almachio quod ego inducias petam ut non urgeat passionem meam. "Cécile leur dit : 'Allez donc dire au malheureux Almachius que je demande une trêve qui relâche son empressement à me faire subir la passion.' »

(4) Raoul de Cluny, Vita Petri Venerabilis 2,10,8: At ille miserabiliter dixit eis quod cuiusdam equi nigri calcibus conculcabatur ita quod nullo modo faciem suam ab eo posset abscondere. "Mais il leur dit sur un ton pitoyable qu'il était foulé sous les talons d'un cheval noir de sorte qu'il ne pouvait d'aucune manière lui dérober son visage. »

Dans un seul cas, à savoir :

(5) Passio Nazarii et Celsi 1,7,3: Altitudine magna absconde nos in terram propter Neronem et ne dixeris amicis tuis quod apud te absconderis nos. "Cache-nous dans un lieu bien haut pour nous dérober à Néron et ne dis pas à tes amis que tu nous tiens cachés chez toi. »

la construction est plus simple du fait de l'absence d'une subordonnée de second degré. En revanche, la régissante est rendue plus complexe par la coordination de deux noyaux prédicatifs : absconde ... et

\footnotetext{
${ }^{8}$ Sur l'emploi de dico quia relevant éventuellement du style mimétique de la bible, voir P. Stotz (1998 : 398).

${ }^{9}$ Voir le dernier tableau en annexe.

${ }^{10}$ Pour le texte de Grégoire le Grand, nous tirons parti du corpus bilingue et reproduisons, pour les exemples cités dans le corps du texte, la traduction disponible en ancien français dans la BFM. Nous commentons cette traduction lorsque la comparaison avec la version latine parait être utile à notre propos. Les traductions en français moderne sont élaborées par nos soins.
} 
ne dixeris. Parfois il y a double complexité et au niveau de la régissante et au niveau de la subordonnée, comme en (2) et (3).

Dico n'est contigu à quod que dans deux cas, à savoir (1) et (2). Dans les autres cas, il en est séparé par le COI / COS désignant le destinataire de la déclaration. À deux reprises, il y a coréférence à la $2^{\mathrm{e}}$ (ex. 5) ou à la $3^{\mathrm{e}}$ personne (ex. 4) entre le sujet de la régissante et celui de la complétive en quod, les agents étant toutefois en (4) distincts du fait de la voix passive du verbe subordonné.

Pour ce qui est de la syntaxe modale, on trouve après quod, de façon sûre, deux fois l'indicatif, deux fois le subjonctif. L'indicatif présent dans l'exemple (1) pourrait s'expliquer par le caractère de vérité générale que revêt l'assertion véhiculée par quod $\mathrm{p}^{11}$. Dans l'exemple (2), le subjonctif plus-queparfait est le mode oblique normalement attendu dans un discours rapporté introduit par un verbe à la $3^{\text {e }}$ personne d'un temps du passé. Pour les mêmes raisons, on attendrait aussi le subjonctif en (4). L'indicatif peut s'y expliquer pragmatiquement par l'aspect de réel que le sujet modal de dixit a voulu conférer au fait rapporté. Cette perception du réel contraste avec celle que présente l'exemple (5), où le subjonctif absconderis semble motivé, à la suite de l'injonction négative ne dixeris, par le souci du locuteur de soustraire à la connaissance d'autrui le contenu de quod $\mathrm{p}$.

En (3) enfin, petam est ambigu : il peut exprimer le futur à l'indicatif ou marquer au subjonctif présent un rapport de simultanéité, voire de postériorité. L'interprétation comme indicatif futur semble toutefois ici, en concordance du présent, la plus plausible ${ }^{12}$ à la suite de l'injonction positive ...et dicite infelici Almachio.

\subsection{2. quia p}

Quia p déclaratif est presque 2,5 fois plus fréquent que quod $\mathrm{p}$. Le verbe dico qui l'introduit peut, comme avec quod $\mathrm{p}$, être accompagné d'un complément au datif précisant le destinataire ${ }^{13}$. Ce qui distingue quia $\mathrm{p}$ de quod $\mathrm{p}$, c'est qu'il s'emploie plus librement en dehors de structures complexes et n'est pas exclu de la position initiale. D'un emploi moins contraint, il semble mieux convenir à la langue spontanée qu'à la langue écrite ${ }^{14}$.

Cela dit, le seul exemple du corpus qui offre une complétive en quia précédant le prédicat recteur, ne le cède en rien en complexité aux énoncés comportant quod $\mathrm{p}$ :

(6) Grégoire, Vita Benedicti 2,15,32 (=80): Quamuis hoc Honoratus eius discipulus cuius mihi relatione conpertum est nequaquam ex ore illius audisse se perhibet sed quia hoc dixerit dictum sibi a fratribus fuisse testatur. "Ia soit ce ke ceste chose Honoreiz ses disciples par cui racontement ge l'ai parceue, la dist soi nient auoir oit de sa boche, mais ke il dist ceste chose il tesmonget ke ce li fut dit des freres. » / « Cependant son disciple Honorât, de qui je tiens l'information, rapporte qu'il n'a pas du tout entendu cette prophétie de sa propre bouche, mais qu'il ait dit cela, ce sont ses frères, selon son témoignage, qui le lui ont assuré. »

La complétive en quia y a probablement été préférée à une proposition infinitive antéposée, de statut thématique, pour la raison qu'elle constitue le sujet d'un verbe au passif, dictum fuisse, qui fait luimême partie d'un A.c.I. Aussi le mode oblique du subjonctif y est-il employé.

La situation des onze exemples comportant une complétive en quia postposée est différente: la structure de l'énoncé y est en général plus directe et transparente. Seulement trois des verbes dépendant de quia figurent au subjonctif. Ces subjonctifs sont confinés dans deux textes rédigés en Italie, à une date assez haute, probablement au $\mathrm{VI}^{\mathrm{e}} \mathrm{s} .{ }^{15}$; employés en concordance du passé au subjonctif imparfait pour exprimer un rapport de simultanéité et appartenant au moins dans deux cas à des passages narratifs, ils peuvent s'expliquer par les règles de l'oratio obliqua:

(7) Passio Pimenii 1,1,13 : Et dum ab omnibus amaretur ipse de se dicebat quia de imperio fuerat procreatus et quia amaretur ab omnibus senatoribus dum elegans videbatur et prudens. «Et alors qu'il était aimé de tous, il disait de lui-même qu'il était de descendance impériale et qu'il était aimé par tous les sénateurs, passant pour un homme distingué et réfléchi. »

\footnotetext{
${ }^{11}$ Pour une explication de l'indicatif dans cet énoncé en rapport avec l'oratio recta, voir infra $§$ 2.1.2.

${ }^{12}$ Cette interprétation est aussi celle retenue par le LASLA.

${ }^{13}$ Quatre exemples dans le corpus : (6), (13), (14) et (15), soit 1/3 des cas contre 2/3 avec quod.

${ }^{14}$ D'où son emploi fréquent, selon P. Greco (2008 : 378), dans le discours direct.

${ }^{15}$ Sur la prépondérance générale de l'indicatif après quia introduisant une complétive avec les uerba sentiendi et dicendi, voir par ex. J. B. Hofmann \& A. Szantyr (1972² : 577) et P. Stotz (1998 : 401-402).
} 
(8) Grégoire, Vita Benedicti 2,3,34 (=61): Quare in me facere ista uoluistis. Numquid non prius dixi quia uestris ac meis moribus non conueniret. " por coi uolsistes uos en moi faire teiz choses ? Nel dis ge dunkes de promiers, ke ne conuenroit pas a mes constumes et az uostres ? / « Pourquoi avez-vous voulu m'infliger ce traitement? $\mathrm{Ne}$ vous ai-je donc pas dit auparavant que cela ne pouvait pas convenir à votre genre de vie ni au mien ?»

(9) Grégoire, Vita Benedicti 2,32,5 (=99) : Cui cum dictum esset quia isdem pater cum fratribus in agro moraretur protinus ante monasterii ianuam corpus extincti filii proiecit et dolore turbatus ad inueniendum uenerabilem patrem sese concitus in cursum dedit. «A cui cant fut dit, ke cil meismes peres demoreuet el champ auoc les freres, enhelement gettat ius deuant l'uiz del monstier lo cors de son mort filh, et il turbleiz de dolor tost soi donat en curs por troueir l'onorable pere. » / «Et comme on lui avait dit que ce même père s'affairait dans le champ avec ses frères, il jeta immédiatement devant la porte du monastère le cadavre de son fils et, égaré de douleur, se mit à courir rapidement à la rencontre du vénérable père. »

En (7), la seconde proposition en quia + subjonctif imparfait est coordonnée à une première comportant un indicatif plus-que-parfait. À la différence modale est donc associée une différence temporelle. L'indicatif plus-que-parfait est employé pour un fait présenté comme objectivement avéré, le subjonctif imparfait pour un sentiment qui ne peut être appréhendé que de façon subjective. Une nuance subjective peut aussi affleurer en (8) pour l'estimation de non-convenance.

Dans les autres exemples à l'indicatif, une lecture de quia $\mathrm{p}$ comme assertive indirecte n'est, dans la plupart des cas, possible qu'au prix d'une infraction à la concordance des temps, la simultanéité par rapport à un temps du passé étant marquée par un indicatif présent au lieu d'un imparfait, l'antériorité par un indicatif parfait au lieu d'un plus-que-parfait :

(10) Passio Caeciliae 1,17,35: Almachius dixit et ego quare non aduerto quo ordine ista prosequaris. Tiburtius dixit quia animalis homo non percipit quae sunt spiritus dei. " Almachius dit : 'Et pourquoi est-ce que moi, je ne me rends pas compte de quelle façon tu parviens à cette constatation ?' Tiburtius dit que l'être humain vivant ne perçoit pas ce qui relève de l'esprit de Dieu. »

(11) Grégoire, Vita Benedicti 2,31,42 (=98) : Ecce est Petre quod dixi quia hii qui omnipotenti deo familiarius seruiunt aliquando mira facere etiam ex potestate possunt. «Voi, ici est, Pirres, ce ke ge dis, ke cil ki seruent al tot poissant deu amiablement, ke il puent a la fie faire merueilhes meismes par poesteit. » / « Voilà bien, Pierre, ce que j'ai dit, à savoir que ceux qui servent avec un dévouement particulier le Dieu tout-puissant peuvent parfois faire des miracles par leur propre pouvoir. »

(12) Grégoire, Vita Benedicti 2,35,45 (=104) : Sed hoc quod dictum est quia ante oculos ipsius quasi sub uno solis radio collectus omnis mundus adductus est sicut numquam expertus sum ita nec conicere scio quoniam quo ordine fieri potest ut mundus omnis ab homine uno uideatur. «Mais ce ke dit est, ke deuant ses oez fut ameneiz toz li monz, alsi com dessuz un rai del soloilh colhiz, alsi com gel unkes n'esprouai, ensi nel sai ge congeteir, par queil ordene puet estre fait, ke toz li monz soit ueuz d'un homme. » / « Mais pour ce qui a été dit que devant ses yeux fut amené le monde entier comme ramassé sous un seul rayon de soleil, si je n'en ai jamais fait l'expérience, je ne puis non plus le conjecturer, car comment se pourrait-il que le monde entier soit vu par un seul homme ?»

(13) Passio Viti 1,4,3 (BHL 8712T) : Sanctus Vitus respondit semel tibi dixi quia Christum filium dii adoro. "Saint Vitus répondit : 'Je t'ai déjà dit une fois que j'adore le Christ, fils de Dieu.' »

(14) Passio Viti 1,4,4 (BHL 8713DT) : At ille nonne inquit iam semel dixi tibi quia Christum filium dei adoro iratusque Valerianus adprehendere ministris iussit infantem. «Mais lui de dire : 'Ne t'ai-je pas déjà dit une fois que j'adore le Christ, fils de Dieu ?'; fâché, Valérien ordonna alors à ses agents de s'emparer de l'enfant. »

Dans (10) et (11), on pourrait certes justifier l'indicatif présent par le caractère gnomique de l'assertion véhiculée par quia $\mathrm{p}$, dans (12), l'indicatif parfait, par l'aspect résultatif d'un accompli au présent que peut impliquer le parfait passif du verbe recteur dictum est. De plus, on pourrait considérer comme des facteurs favorisant l'emploi de quia p au détriment de l'A.c.I. dans (11) et (12) la relation cataphorique qui s'instaure de la régissante à la subordonnée complétive ${ }^{16}$, jointe en (12) à la voix

\footnotetext{
16 Si la diaphore passe normalement pour un facteur favorisant l'emploi de quod p (Cuzzolin 1994a : 208 ; Bodelot 2000 : 73-83), elle a pu jouer aussi un rôle important dans le passage de quod relatif à quod complétif (Mayen 1889 : 5-12 ; 28-29; Serbat $2003: 741$ ).
} 
passive du verbe introducteur; dans (10), on pourrait enfin mettre en avant la nature ambiguë de quia, qui, à la suite de quare interrogatif, peut encore être compris comme une conjonction causale («parce que ») introduisant une proposition circonstancielle. On y a affaire à un de ces contextes «critiques » (Diewald 2002 : 109-114) qui ont dû médiatiser le passage de quia «parce que » à quia « que ». Ainsi, on peut y admettre, sans différence appréciable du sens global de l'énoncé, une lecture de quia $\mathrm{p}$ comme complément essentiel de dixit ou comme complément périphérique d'une proposition implicite du type de : Tiburtius dixit : '<Non aduertis quo ordine ista prosequar $>$ quia...' .

Toutefois, une autre interprétation possible est de voir dans les propositions en quia citées dans (10) à (14) et aussi dans la proposition en quod citée en (1) des instances de discours direct, les coordonnées déictiques, y inclus les temps verbaux de quia ou quod $\mathrm{p}$, se définissant par rapport au hic et nunc du locuteur originel. Y verra-t-on simplement l'indice d'une perméabilité des frontières entre discours direct et discours indirect ${ }^{17}$ ou ira-t-on jusqu'à y considérer quia et quod ${ }^{18}$ comme des introducteurs de discours direct? Ce cas de figure s'inscrirait dans le cadre très riche et prolifique des assertives directes attestées en grand nombre à la suite du verbe dicere dans le corpus. L'affectation de quia et quod à l'introduction d'une citation ou d'un discours direct en latin tardif à partir de l'Itala est signalée par différents grammairiens ${ }^{19}$. On y voit normalement une influence du ö $\tau$ recitatiuum grec (Stotz 1998 : 403). Une étude récente de ö $\tau$ introduisant le discours direct dans le Nouveau Testament (Viellard : 2013) rejette l'hypothèse d'un hébraïsme ou d'une simple marque de niveau de langue familier. L'emploi de ö ıı répondrait à un parti pris du locuteur primaire, qui, par ce biais, essaie de focaliser et de solenniser les propos rapportés : ötı correspondrait à une marque de vérité du discours, que Luther, dans sa traduction de la bible, a souvent transposée par all. « doch». Cette interprétation pragmatique peut convenir dans notre corpus au caractère assertif fort dont sont normalement dotées les instances de discours en question ${ }^{20}$. Comme en (13) et (14), on a encore affaire en (15) à une fervente profession de foi qui paraît être directement rapportée ou citée :

(15) Passio Ansani et Maximae 1,3,11: Beatus namque martyr coepit meditari infra semet ipsum dicens uadam et dicam imperatoribus quia christianus sum et pro illius nomine mori desidero. " Et de fait le bienheureux martyr se mit à réfléchir en son for intérieur et dit : 'J'irai dire aux empereurs que je suis chrétien et que je désire mourir en son nom.' »

Que le locuteur actuel coïncide de préférence avec le locuteur originel représente un autre indice défavorable à l'interprétation des paroles rapportées comme véritable discours indirect (Greco 2015). Comme cet emploi survit en roman, une évolution en partie propre au latin n'est pas exclue ${ }^{21}$. Dans le corpus hagiographique, les occurrences de quod/quia se prêtant à une telle lecture sont confinées dans les textes rédigés en Italie ${ }^{22}$. On y reconnait en général un trait mimétique du latin chrétien (Stotz 1998 : 404). Notons que, dans les trois exemples tirés de la Vita Benedicti de Grégoire, (1), (11) et (12), quod / quia latin est maintenu en ancien français et transposé par «ke».

Si l'on admet cet emploi de quia dit recitatiuum dans les exemples suscités, il ne reste finalement qu'un seul emploi qui mérite d'être commenté :

(16) Passio Caeciliae 1,30,15 : Caecilia respondit dixisti principes tuos uiuificandi et mortificandi tibi copiam tribuisse cum solam mortificandi scias tibi traditam potestatem uitam enim uiuentibus tollere

\footnotetext{
${ }^{17}$ Sur le glissement du discours indirect vers le discours direct et vice-versa, voir P. Greco (2012:69-70) et C. Bodelot (2014 : 189 ; à paraître a).

${ }^{18}$ Voir l'exemple cité en (1).

${ }^{19}$ Voir par ex. les développements chez J. B. Hofmann \& A. Szantyr (1972² : 578) et P. Stotz (1998 : 403-405).

20 On pourra parler dans ce cas d'une "pragmaticalisation» de ö $\tau$, dans la mesure où une unité lexicale / grammaticale développe un emploi « où elle ne joue pas un rôle sur le plan référentiel, mais bien sur le plan conversationnel » (Dostie 2004 : 27). Aussi sa portée n'est-elle pas intraphrastique : elle appartient à la macro-syntaxe du discours (ibid. : 30, 44).

${ }^{21}$ Voir J. B. Hofmann \& A. Szantyr (1972² : 578-579), avec références bibliographiques.

${ }^{22}$ Nous faisons ici abstraction de Raoul de Cluny, Vita Petri Venerabilis 2,7,22: Raptus enim fuerat ad iudicium et ei insistebat turba daemonum eum fortiter accusantium et dicentium quia haec fecit et merito debet nobiscum reputari, où quia est, à notre sens, à l'intérieur d'une instance de discours direct, susceptible d'une lecture causale, si l'on interprète le dernier et comme l'équivalent de etiam: "Il avait été traîné devant le tribunal et là une foule de démons se mettait à ses trousses l'accusant avec véhémence et disant : 'Parce qu'il a fait cela, il doit aussi à juste titre être mis en examen en notre présence.' »
} 
potes mortuis uitam dare non potes dic ergo quia imperatores tui mortis ministrum te esse uoluerunt. "Cécile répondit : 'Tu as dit que tes maîtres t'avaient accordé la faculté de faire vivre et de faire mourir alors que tu sais que seul le pouvoir de faire mourir t'a été attribué ; tu peux en effet enlever la vie aux vivants, tu ne peux pas donner la vie aux morts. Dis donc que tes empereurs ont voulu que tu sois le serviteur de la mort.' »

Le possessif tui, qui est coréférentiel avec le sujet modal de dic, exclut toute possibilité d'interpréter quia $\mathrm{p}$ comme une instance de discours direct. Le recours à la subordination explicite semble ici motivé par la modalité impérative du verbe introducteur ${ }^{23}$, qui, imposant une lecture fortement assertive du contenu de $\mathrm{p}$, passe en général pour un facteur défavorable au maintien de l'A.c.I. Cette valeur déclarative forte est ici corroborée par l'emploi de l'indicatif uoluerunt ${ }^{24}$, qui exprime le haut degré de vérité conféré par Caecilia au contenu de la complétive.

On n'a repéré dans le corpus, dans le sillage de dicere, aucun exemple d'une déclarative introduite par quoniam. Mais quoniam p complétif est attesté avec d'autres verbes introducteurs ${ }^{25}$.

\subsection{3. ut $\mathrm{p}$}

Le corpus offre, dans le contexte de dico, un exemple d'une subordonnée en $u t+$ subjonctif susceptible d'une interprétation déclarative :

(17) Passio Nazarii et Celsi 1,1,23: Hoc audito Affricanus pater eius cum tristitia dixit ei fili Nazari in omni dignitate mea contendo ut uiuas mihi annis multis et deum colas meo more et excites mihi semen de uxore quam dabo tibi cum coronis et choris cum gaudio totius Romae et tu dicis mihi ut discas baptismum Petri propter quod occisus est. « Après avoir entendu cela, son père Africanus lui dit avec tristesse : 'Mon fils, Nazaire, c'est pour moi un point d'honneur de t'engager à vivre près de moi de longues années, à vénérer Dieu selon ma coutume et à me créer une descendance avec une femme que je te donnerai sans lésiner sur couronnes et danses, au milieu d'une grande allégresse pour la Rome tout entière, et toi, tu me dis que tu te prépares au baptême de Pierre, qui a provoqué sa mort.' »

Cette interprétation s'oppose de façon significative à la lecture jussive, courante, qu'admet dico ut $\mathrm{p}$ en :

(18) Passio Agathae 1,10,7: Tunc dicebant sanctae Agathae personae quae ibi erant inclusae ut abiret. «

«Alors les personnes qui y étaient enfermées disaient à sainte Agathe de partir. »

(19) Grégoire, Vita Benedicti 2,17,16 (= 93) : Quos uir dei deferri iussit et adflicto petitori tribuit dicens ut duodecim redderet et unum in expensis propriis haberet. "Les queiz li hom deu ke hom li portast comandat, et il les donat al afflit proior disanz, ke il rendist les doze et l'un auroit en ses propres despenses. » / «L'homme de Dieu ordonna de les (scil. les écus d'or) lui apporter et les donna au débiteur désespéré lui disant d'en rendre douze et d'en garder un pour couvrir ses propres dépenses. »

C'est donc ici le contexte qui oriente $u t \mathrm{p}$ vers une force illocutoire assertive ou jussive. L'emploi assertif de $u t \mathrm{p}$ dans le sillage d'un verbe de déclaration se trouve attesté dans d'autres textes tardifs ${ }^{26}$. Que cet usage se rencontre dans notre corpus dans la Passio Nazarii et Celsi est peut-être à mettre en relation avec l'absence totale d'A.c.I. intervenant dans ce texte en séquence avec dico ${ }^{27}$. Par rapport à quod / quia, notamment suivi de l'indicatif mais aussi du subjonctif, $u t+$ subjonctif peut être l'indice

\footnotetext{
${ }^{23}$ Voir aussi P. Greco (2008: 376).

${ }^{24}$ Voir supra notre explication contrastive du subjonctif employé dans quod p à la suite de ne dixeris dans (5). Dans le cadre de l'A.c.I., une telle opposition significative des modes verbaux serait évidemment impossible.

${ }^{25}$ Voir par ex. Passio Caeciliae 1,30,1 : infelix ignoras quoniam mortificandi et uiuificandi mihi ab inuictissimis principibus potestas est data. "'malheureuse, tu ignores que le pouvoir de faire mourir et de faire vivre m'a été donné par nos maîtres qui n'ont encore absolument jamais été vaincus.' »; Passio Viti 1,16,9 (BHL 8711T) : ... tibi promitto quoniam saluus eris. « ... je te promets que tu seras sain et sauf. »

${ }^{26}$ Pour des exemples, voir par ex. J. Herman $(1963: 46)$.

${ }^{27}$ À ce propos, voir aussi infra. P. Stotz (1998: 399) considère l'emploi de ut $\mathrm{p}$ à la place de l'A.c.I. comme un hyperurbanisme, contrastant avec l'emploi populaire de quod / quia.
} 
d'une assertivité réduite dans la mesure où le locuteur actuel désapprouve le contenu des propos rapportés du locuteur originel et s'en distancie ${ }^{28}$.

Au départ, la substitution de $u t \mathrm{p}$ à une infinitive déclarative a pu être déclenchée par la limite flottante qui existe dans certaines situations entre la force illocutoire véhiculée par l'A.c.I. et celle véhiculée par $u t+$ subjonctif. Ainsi, en latin classique, avec dico le sens injonctif a pu être exprimé par $u t+$ subjonctif ou par une infinitive comportant un verbe modal du type de debere. D'où probablement l'impression que $u t \mathrm{p}$ et l'A.c.I. étaient interchangeables dans de nombreux contextes, y inclus des contextes simplement assertifs ${ }^{29}$.

À notre sens, une autre source de confusion interne au latin a encore pu être constituée par l'emploi très fréquent de $u t$ comme adverbe de manière introduisant une interrogation indirecte (Bodelot 1990 : $16-17$; 29 ; 38-39; 44). Vt, se vidant de son sens de manière («comment ») ${ }^{30}$, a pu, par grammaticalisation, passer du statut d'adverbe interrogatif à celui de conjonction assertive, sans hypothéquer davantage la vérité de la prédication subordonnée qu'il ne le fait dans une interrogative dépendant d'un uerbum dicendi $i^{31}$.

\subsection{Les propositions infinitives}

Pour ce qui est des déclaratives indirectes à l'A.c.I., on relève d'emblée une différence notable, d'ordre syntagmatique, par rapport aux déclaratives conjonctionnelles. Sur 54 séquences à l'infinitif, 17 , donc presque $1 / 3$, sont placées dans la chaîne avant le verbe dicere, qui figure toujours à la voix active et n'est jamais accompagné d'un datif exprimant le destinataire. On parlera dans la suite d'A.c.I. antéposé au cas où la proposition infinitive précède en bloc la proposition régissante comme en (20) ou - cas plus fréquent - est imbriquée dans la régissante comme en (21), mais toujours de façon que le verbe dico suit immédiatement l'A.c.I., normalement le verbe à l'infinitif :

(20) Raoul de Cluny, Vita Petri Venerabilis 2,3,11 : [Praesentia nihil esse] dicebat ... « Il disait que les biens présents ne valaient rien ...»

(21) Passio Marcelli et Apuleii 1,7,9 : ... clara uoce [christianum me esse] dixi ... «... j'ai dit haut et fort que j'étais chrétien ...»

Cette position frontale avant le verbe recteur semble être tellement exclusive de la proposition infinitive qu'il n'y a pas lieu de rechercher des facteurs morphologiques ou syntaxiques favorables à sa non-concurrence avec quod $\mathrm{p}$ ou quia $\mathrm{p}$.

Souvent ce sont des facteurs pragmatiques, tel le statut thématique de la déclarative indirecte qui implique en latin son avancée dans la phrase, et partant sa mise en forme infinitive. À preuve l'extrait suivant de Grégoire, où le fait d' «être avec soi » constitue le topos du passage :

(22) Grégoire, Vita Benedicti 2,3,48-63 (=62) : Nam quotiens per cogitationis motum nimiae extra nos ducimur et nos sumus et nobiscum non sumus quia nosmet ipsos minime uidentes per alia uagamur. An illum secum fuisse dicimus qui in longinquam regionem abiit portionem quam acceperat consumpsit uni in ea ciuium adhaesit porcos pauit quos et manducare siliquas uideret et esuriret. Qui tamen cum postmodum coepit cogitare bona quae perdidit scriptum de illo est in se reuersus dixit quanti mercenarii in domo patris mei abundant panibus si igitur secum fuit unde ad se rediit. Hunc ergo uenerabilem uirum secum habitasse dixerim quia in sua semper custodia circumspectus ante oculos conditoris se semper aspiciens se semper examinans extra se mentis suae oculum non deuulgauit. "Quar quantes fies par lo mouement de la pense trop sumes meneit defors nos meismes, et nos sumes et auoc nos ne sumes nos mie, quar nient ueant nos meismes, par altres choses uaions. Disons nos dunkes celui auoir esteit auoc soi, ki s'en alat en une lointaine contreie, ki deguastat la parzon cui il auoit prise, ki aerst en cele contreie a un des citains, ki paut les pors les queiz il uerroit

\footnotetext{
${ }^{28}$ Sur l'incidence possible, en latin mérovingien, du degré de commitment du locuteur actuel et / ou originel sur le choix de quod / quia ou ut $\mathrm{p}$ en dépendance d'un uerbum dicendi ou sentiendi, voir P. Greco (2014).

${ }_{29}$ Pour cette explication et d'autres hypothèses concernant l'origine de l'emploi déclaratif de $u t \mathrm{p}$, voir J. Herman (1963 : 46-47) et R. Sornicola (2014 : 362-366).

${ }^{30}$ Dans le corpus, $u t$ est encore attesté avec ce sens interrogatif chez Sulpice Sévère, dans l'exemple (59).

${ }^{31}$ Que la même ambiguité existe dans certains contextes pour quomodo ou qualiter, qui peuvent aussi s'interpréter comme adverbes interrogatifs ou conjonctions introduisant une assertive, on le verra au § 3.2.1. Si nous avons choisi de ne pas traiter ces exemples sous la présente rubrique des déclaratives indirectes, c'est que, synchroniquement, on peut dans ce cas encore hésiter entre l'interprétation interrogative et l'interprétation assertive.
} 
mangier les leguns et si auroit fain ? Li queiz nekedent quant il en apres comenzat a penseir les biens cui il perdit, si est escrit de lui : Il retorneiz en soi dist : Quant lowiz en la maison de mon pere sont raemplit de pains ! Gieres se il astoit auoc soi, dont repairat il a soi ? Por ice ai ge dit cest honorable baron auoir meis auoc soi, car il toz tens porueuz en sa garde, deuant les oez de son faiteor toz tens soi regardanz, toz tens soi porpensanz, defors soi ne depuliat mie l'oelh de sa pense. » / «Car chaque fois que sous l'impulsion d'une pensée excessive nous sommes jetés en dehors de nous, tout en étant nous, nous ne sommes pas avec nous parce que, ne nous voyant nullement nous-mêmes, nous nous projetons sur d'autres objets. Ou disons-nous qu'a été avec lui celui qui est parti dans une contrée lointaine, a dévoré la portion qu'il avait reçue, s'est attaché dans cette contrée à un citoyen, a fait paître ses pourceaux qu'il voyait manger des légumes tandis que lui-même avait faim. Mais quand plus tard il pensa aux biens qu'il avait perdus, revenu à lui, il dit, comme on le lit dans la Sainte Écriture: 'Que de mercenaires vivent dans la maison de mon père qui ont du pain en abondance !' Donc s'il a été avec lui, d'où est-il revenu à lui ? Je dirais alors que ce vénérable anachorète a demeuré avec lui parce que, veillant toujours sur lui-même dans sa cellule, se considérant toujours et s'examinant toujours sous le regard de son Créateur, il ne divulgua jamais ce que percevait sa pensée. »

On remarque que, dans la transposition en français ancien aussi bien que moderne, le verbe « dire » précède à deux reprises la complétive déclarative ; en ancien français, sur le modèle du latin, il engendre, dans l'un et l'autre cas, encore une proposition infinitive.

Ailleurs, c'est pour des raisons de focalisation qu'une infinitive occupe en latin la position initiale de la phrase. Ainsi encore, chez Grégoire, dans l'exemple (23), où, par souci de contraste, le contenu de la première infinitive est, du fait de son antéposition, davantage mis en exergue que celui de la seconde, postposée à dicebat. Non aléatoire, l'emplacement distinct des deux infinitives est au service d'un style contrôlé ; il doit ici refléter l'humble modestie de Maur :

(23) Grégoire, Vita Benedicti 2,7,20-26 (= 68-69) : Reuersus ad patrem rem gestam retulit. Vir autem uenerabilis Benedictus hoc non suis meritis sed oboedientiae illius deputare coepit. At contra Maurus pro solo eius imperio factum dicebat seque conscium in illa uirtute non esse quam nesciens fecisset. Sed in hac humilitatis mutuae amica contentione accessit arbiter puer qui ereptus est. " Il soi retornat a son pere, si racontat la chose ki faite astoit. Et li honorables hom Benoiz comenzat ceste chose a raconteir nient a ses desertes, mais a l'obedience de celui. Mais la encontre Mors disoit ce estre fait por soul lo sien comant, et soi nient estre sachable en icele uertut cui il eust faite nient sachanz. Mais en ceste amiable tenzon d'entrechaniable humiliteit nient li enfes iugieres ki fut deliureiz. » / « Revenu auprès du père, il lui rapporta l'exploit. Mais le vénérable Benoît se mit à l'imputer non à ses propres mérites mais à l'obéissance de son interlocuteur. Maur, par contre, disait que cela n'avait été accompli qu'en vertu de son commandement et qu'il n'était pas responsable de ce miracle qu'il avait fait sans le savoir. Mais au milieu de ce concours amical d'une humilité réciproque, l'enfant arraché aux flots survint comme arbitre. »

En français, le verbe de parole précède les deux complétives; les propositions infinitives sont en ancien français à nouveau calquées sur le latin, y compris le sujet de l'infinitive « soi », qui transpose le réfléchi indirect du latin se. On a dans ce cas affaire en ancien français à une subordination complétive savante, caractéristique de la langue vernaculaire précoce de traduction.

Grégoire est le seul auteur du corpus à offrir plus d'infinitives (6 contre 4) antéposées que postposées à dicere. Ces séquences à l'infinitif sont - nous l'avons vu - dans le corpus dans $2 / 3$ de leurs emplois (37 sur 54, soit $68,5 \%)^{32}$ postposées au verbe recteur. Cette représentation prépondérante dans une position que pourraient aussi occuper des propositions conjonctionnelles explique, en partie au moins, la fréquence très faible de ces dernières dans le corpus.

Parmi les rares facteurs morphosyntaxiques défavorables à l'emploi d'une infinitive postposée, on peut citer la voix passive du verbe dicere, qui engendre plutôt une déclarative conjonctionnelle ${ }^{33}$. Un

\footnotetext{
32 Nous comptons ici en «séquences». Si l'on compte séparément toutes les infinitives, mêmes celles coordonnées et dépendant d'un seul et même verbe introducteur, ce chiffre s'élève à $73 \%$ (46 unités sur 63) ; comparer le tableau 1, qui fait état d' « unités », avec le tableau en annexe, qui opère un double compte en « séquences » et en « unités ».

${ }^{33}$ Voir les exemples de Grégoire cités en (6), (9) et (12).
} 
seul exemple de ce type a été repéré dans le corpus ; 1'A.c.I. y est maintenu à la suite d'un adjectif verbal employé à la forme passive :

(24) Passio Viti 1,2,11 (BHL 8711T): Hylas dixit quantum ad rem spectat dicendum est potius esse supplicium quod confiteris fore mysterium. «Hylas dit: 'En ce qui concerne cette affaire, il faut dire qu'est plutôt un supplice ce qui, selon tes révélations, serait un mystère.' »

Ici, la présence d'une relative en quod a dû dissuader l'auteur d'employer à peu de distance une complétive en quod/quia. De plus, il existe sur le plan stylistique un parallélisme entre les deux infinitives esse supplicium et fore mysterium, le dernier A.c.I. ne pouvant d'ailleurs guère commuter avec une conjonctionnelle en raison du pronom relatif à l'accusatif quod qui introduit l'un de ses deux constituants.

Aussi est-ce dans le contexte d'une relative que l'infinitive est particulièrement bien représentée ${ }^{34}$. Si le verbe dico, engendrant à sa suite une séquence infinitive, ne se présente, d'une façon générale, que dans $37,8 \%$ des cas $(14 \text { cas sur } 37)^{35}$ sous forme indépendante, le cadre préféré dans lequel il intervient en construction subordonnée est la relative.

Tableau 2 :

Niveau d'enchâssement de la séquence complétive

\begin{tabular}{|l|l|l|l|l|l|l|}
\hline & \multicolumn{2}{|c|}{ A.c.I. } & \multicolumn{2}{c|}{ quia $p$} & \multicolumn{1}{c|}{ quod $p$} & \multicolumn{1}{c|}{ ut $p$} \\
\hline & antéposé & postposé & antéposé & postposé & postposé & postposé \\
\hline 1er niveau & $13(76,5 \%)$ & $\mathbf{1 4}(\mathbf{3 7 , 8} \%)$ & $0(0 \%)$ & $7(70 \%)$ & $4(80 \%)$ & $1(100 \%)$ \\
\hline 2e niveau & $4(23,5 \%)$ & $17(46 \%)^{*}$ & $1(100 \%)$ & $3(30 \%)$ & $1(20 \%)^{* *}$ & $0(0 \%)$ \\
\hline 3e niveau & $0(0 \%)$ & $6(16,2)^{* *}$ & $0(0 \%)$ & $0(0 \%)$ & $0(0 \%)$ & $0(0 \%)$ \\
\hline Total & $17(100 \%)$ & $37(100 \%)$ & $1(100 \%)$ & $10(100 \%)$ & $5(100 \%)$ & $1(100 \%)$ \\
\hline
\end{tabular}

*Six de ces séquences à l'A.c.I. dépendent de dicere figurant au participe présent et considéré pour cette raison comme subordonné.

** Une de ces séquences à l'A.c.I. dépend de dicere figurant au participe présent et considéré pour cette raison comme subordonné.

*** Cette séquence quod $\mathrm{p}$ dépend de dicere figurant au participe présent et considéré pour cette raison comme subordonné.

$\mathrm{Si}$ avec un pronom relatif sujet du seul verbe dicere, l'A.c.I. pourrait commuter en (25) avec une conjonctionnelle en quod ou quia:

(25) Passio Agathae 1,13,6 : ... nec postea eum aliquis uidit nec inuentus est aliquis qui diceret hunc se scire . «... et plus tard personne ne le vit et il ne se trouva personne pour dire qu'il le connaissait. »

une telle commutation n'est guère concevable lorsque le pronom relatif est partie prenante dans la construction infinitive. Suite à la «montée » obligatoire du thème en $q u$-relatif ou interrogatif ${ }^{36}$, le pronom relatif est forcément extraposé, l'ordre des mots normal étant alors 'pronom relatif + dicere antéposé à l'infinitif' ${ }^{37}$ :

\footnotetext{
${ }^{34}$ On pourrait d'ailleurs faire la même remarque au sujet du français de la fin du moyen français et du français préclassique, ce qui montre que les latinismes syntaxiques étaient bien acceptés par le système du français.

${ }^{35} \mathrm{Si}$ l'on inclut dans le compte des verbes dicere subordonnés aussi les participes, il ne reste en effet sur les 37 occurrences de dico que 14 emplois indépendants ; les 23 emplois subordonnés de dicere se répartissent comme suit : 7 emplois participiaux, 16 emplois en proposition subordonnée, dont 9 en subordonnée relative et 7 dans une circonstancielle ( 4 en $u t$ final ou consécutif ; 2 en cum ; 1 en ac si).

${ }^{36}$ Sur le wh-movement, voir par ex. N. Chomsky (1977: 71-132).

${ }^{37}$ Dans deux cas seulement, on a la séquence suivante : qu-interrogatif ou relatif + infinitif (éventuellement sous-entendu s'il doit revêtir la forme de esse) + dicere (postposé) ; voir Grégoire, Vita Benedicti 2,38,13 (= 107) : Quidnam esse dicimus quod plerumque in ipsis quoque patrociniis martyrum sic esse sentimus ut non ... ; «Que dire donc de la raison pour laquelle / du fait que, la plupart du temps lorsque nous implorons la protection des martyrs, nous nous rendons compte qu'il arrive que ne ... pas ... ?»; Adson de Montier-en-Der, Vita
} 
(26) Sulpice Sévère, Vita Martini 1,13,3: si habes inquit aliquam de deo tuo quem dicis te colere fiduciam ... (* quem dicis quod/quia colis) «'si tu as, dit-il, quelque confiance en ton Dieu que tu prétends vénérer...'»

(27) Passio Saturnini 1,7,6: Quomodo uultis ut ego eos timeam a quibus ut audio dicitis me timeri. (? a quibus ... dicitis quod/quia timeor) «'Comment voulez-vous que je craigne ceux dont vous dites, à ce que j'entends, qu'ils me craignent?' »

En français usuel, pareille imbrication donne normalement lieu à une désynthétisation constructionnelle, que rend possible l'emploi d'une subordonnée déclarative explicite ${ }^{38}$ :

$(26$ ') « ' $\ldots$ ton Dieu dont tu dis que tu le vénères' »

$\left(27\right.$ ') ? « '... ceux par qui / dont vous dites ... que je suis craint' $\left.»^{39}\right\rangle$ «'... ceux dont vous dites ... qu'ils me craignent' »

Mais ce qui frappe surtout dans le corpus, c'est que l'infinitive se rencontre aussi dans des contextes syntaxiques qui passent en général pour peu propices à son maintien, à savoir des énoncés longs et complexes qui peuvent donner lieu à des structures opaques (Calboli 1987 : 25 sqq.). Ainsi, le tableau 3 montre que, sur les 46 propositions infinitives postposées intégralement ou partiellement à 37 verbes dicere, seulement $13(=28,3 \%)$ interviennent en structure simple, non coordonnées à une ou plusieurs autres infinitives ou sans qu'il y ait une subordonnée de second degré qui soit imbriquée dans l'infinitive ou y soit attachée en remorque. Sur ce point, l'A.c.I. postposé ne le cède qu'à quod p, qui n'intervient que dans $20 \%$ ( 1 cas sur 5) en structure simple. Les énoncés complexes sont plus fréquents avec un A.c.I. postposé qu'avec un A.c.I. antéposé puisque, dans ce dernier cas, à peu près la moitié des exemples ( 8 sur 17) présentent une structure simple et transparente.

Tableau 3:

Constitution de la structure subordonnée

\begin{tabular}{|l|l|l|l|l|l|l|}
\hline & \multicolumn{2}{|c|}{ A.c.I. } & \multicolumn{2}{c|}{ quia $p$} & quod $p$ & \multicolumn{1}{c|}{ ut $p$} \\
\hline & antéposé & postposé & antéposé & postposé & postposé & postposé \\
\hline complétive non co-/superordonnée à d'autres $p$ & $8(47 \%)$ & $\mathbf{1 3 ( 2 8 , 3 \% )}$ & $1(100 \%)$ & $7(63,6 \%)$ & $\mathbf{1 ( 2 0} \%)$ & $0(0 \%)$ \\
\hline complétive co-/superordonnée à d'autres $p$ & $9(53 \%)$ & $33(71,7 \%)$ & $0(0 \%)$ & $4(36,4 \%)$ & $4(80 \%)$ & $1(100 \%)$ \\
\hline Total & $17(100 \%)$ & $46(100 \%)$ & $1(100 \%)$ & $11(100 \%)$ & $5(100 \%)$ & $1(100 \%)$ \\
\hline
\end{tabular}

On ne trouve d'ailleurs jamais en position frontale une suite de propositions infinitives coordonnées, alors que ce cas de figure se présente une dizaine de fois en cas de postposition ${ }^{40}$.

De plus, la proposition infinitive postposée est susceptible de descendre plus bas dans la structure hiérarchique de la phrase que les autres complétives. Elle est la seule à constituer - et cela à six reprises - une subordonnée de troisième degré ${ }^{41}$.

Pour l'A.c.I. postposé, citons à titre d'illustration quelques exemples particulièrement complexes $^{42}$ :

Walberti 2,7,24 : Inter que uicum quoque quem superius Nant uocatum diximus ... «Et au milieu du village dont nous avons dit plus haut qu'il s'appelait Nant ... ».

${ }^{38}$ Il faut peut-être mettre à part certaines constructions simples, où le relatif représente par ex. l'agent d'une infinitive avec verbe intransitif ; dans ce cas, l'extraction semble beaucoup plus acceptable, et le français a connu la possibilité de tours comme : «X, qu'on dit être venu ».

${ }^{39}$ Même avec une subordonnée déclarative explicite en français, il semble difficile de maintenir la construction avec verbe conjugué au passif « que je suis craint » pour reproduire l'infinitive passive du latin me timeri.

${ }^{40}$ Voir par ex. Raoul de Cluny, Vita Petri Venerabilis 2,16,8-11 : Dicebat enim rusticus serpentem corpus suum intrasse et hac occasione se assidue torqueri et per singula momenta proximum morti fieri. « Le campagnard disait en effet qu'un serpent avait envahi son corps, que de ce fait il était continuellement mis à l'épreuve et que par moments il frôlait la mort. » Cela explique qu'on ait inventorié pour 37 verbes dicere introducteurs 46 A.c.I. postposés (répartis en 37 « séquences » infinitives).

${ }^{41}$ Voir le tableau 2.

${ }^{42}$ Voir aussi l'exemple (24) commenté supra, où le sujet de l'infinitive dépendant de dicendum est est constitué d'une relative imbriquée dans une autre infinitive, ainsi que les exemples (26) et (27) avec relative imbriquée, où la proposition infinitive constitue une subordonnée de troisième degré. 
(28) Passio Agnetis 1,6,10: Tunc quidam extitit ex parasitis eius qui diceret hanc christianam esse ab infantia et magicis artibus ita occupatam ut Christum dicat sponsum suum. (Deux infinitives coordonnées interviennent à un second niveau d'enchâssement; la seconde prend en remorque une circonstancielle consécutive) «Alors l'un de ses parasites se présenta pour dire qu'elle était chrétienne depuis son enfance et tellement adonnée aux pratiques magiques qu'elle affirmait que le Christ était son fiancé. »

(29) Passio Caeciliae 1,12,9: ... certe unum deum dicitis esse in caelis credendum. (Deux infinitives, imbriquées l'une dans l'autre, interviennent à deux niveaux hiérarchiques différents) «... vous dites avec conviction qu'il faut croire qu'un seul dieu est aux cieux. »

(30) Sulpice Sévère, Vita Martini 1,16,5: Qua ille uoce confusus obstipuit et refugit dicens hoc suae non esse uirtutis senem errare iudicio non esse se dignum per quem dominus signum uirtutis ostenderet. (Trois infinitives, intervenant à un second niveau d'enchâssement, sont juxtaposées ; la dernière intègre une relative) «Confondu par ces paroles, il resta interdit et recula en disant qu'il n'avait pas ce pouvoir, que le vieillard se trompait et qu'il n'était pas digne que le Seigneur se serve de lui pour donner une preuve de son pouvoir. »

(31) Grégoire, Vita Benedicti 2,3,102 (=64) : Numquidnam Paulum mortem dicimus timuisse quam se ipse pro Iesu amore testatur appetere. (Une première infinitive comporte un COD intégrant une relative qui engendre elle-même une infinitive dont le COD est constitué du relatif) ${ }^{43}$ " Disons nos dunkes Paulum auoir cremut la mort, cui il meismes tesmonget por l'amor de Ihesu soi desireir ?» / « Disons-nous donc que Paul a redouté la mort à laquelle il prétend lui-même aspirer par amour de Jésus?»

Que l'A.c.I. soit antéposé ou postposé, il intervient le plus souvent en concordance du passé, de préférence pour exprimer un rapport de simultanéité :

Tableau 4 :

Concordance des temps dans les propositions infinitives

\begin{tabular}{|l|l|l|l|}
\hline Sphère temporelle & Rapport des temps & A.c.l. antéposé & A.c.l. postposé \\
\hline au présent & simultanéité & $2(11,8 \%)$ & $12(26,1 \%)$ \\
\hline au présent & antériorité & $2(11,8 \%)$ & $1(2,2 \%)$ \\
\hline au présent & postériorité & $1(5,9 \%)$ & $0(0 \%)$ \\
\hline au passé & simultanéité & $\mathbf{7 ( 4 1 , 1 \% )}$ & $\mathbf{2 4 ( 5 2 , 2 \% )}$ \\
\hline au passé & antériorité & $5(29,4 \%)$ & $9(19,5 \%)$ \\
\hline au passé & postériorité & $0(0 \%)$ & $0(0 \%)$ \\
\hline Total & & $17(100 \%)$ & $46(100 \%)$ \\
\hline
\end{tabular}

* Selon les marques formelles.

Un trait significatif de dico précédant l'A.c.I. est qu'il figure dans $59,5 \%$ des cas (22 séquences sur $37)$ à la $3^{\mathrm{e}}$ personne, dans une faible proportion $(13,5 \%$, c.-à-d. 5 séquences sur 37$)$ à la $1^{\text {ère }}$ personne. Cette faible représentation de la $1^{\text {ère }}$ personne contraste avec ce qu'on constate pour l'A.c.I. antéposé et surtout pour quia p postposé, où le verbe dicere figure respectivement dans $35,3 \%$ (6 séquences sur 17 ) et $50 \%$ (5 séquences sur 10$)$ à la $1^{\text {ère }}$ personne :

Tableau 5 :

Référence personnelle du verbe recteur dicere

\begin{tabular}{|l|l|l|l|l|}
\hline & V 1ère pers. & V 2e pers. & V 3e pers. & Total \\
\hline avec A.c.I. antéposé(s) & $\mathbf{6}(\mathbf{3 5 , 3} \%)$ & $2(11,8 \%)$ & $9(52,9 \%)$ & $17(100 \%)$ \\
\hline avec A.c.l. postposé(s) & $5(13,5 \%)$ & $10(27 \%)$ & $\mathbf{2 2}(59,5 \%)$ & $37(100 \%)$ \\
\hline avec quia p antéposé(s) & $0(0 \%)$ & $0(0 \%)$ & $1(100 \%)$ & $1(100 \%)$ \\
\hline avec quia p postposé(s) & $5(50 \%)$ & $1(10 \%)$ & $4(40 \%)$ & $10(100 \%)$ \\
\hline avec quod p postposé(s) & $1(20 \%)$ & $2(40 \%)$ & $2(40 \%)$ & $5(100 \%)$ \\
\hline avec ut p postposé(s) & $0(0 \%)$ & $1(100 \%)$ & $0(0 \%)$ & $1(100 \%)$ \\
\hline
\end{tabular}

\footnotetext{
${ }^{43}$ Comme en (23), les infinitives de l'ancien français sont calquées sur le latin, tout comme « soi », qui reproduit le réfléchi indirect se du latin.
} 
La référence plus fréquente du verbe dicere à la 1 ère personne peut dans le cas de quia $\mathrm{p}$ s'expliquer par la forte assertivité de la construction et son affinité avec l'oratio recta, dans le cas de l'infinitive antéposée, par sa vertu souvent focalisante. Dans le cas de l'A.c.I. (ou au moins de l'infinitif) postposé, le verbe dico, figurant de préférence à la $3^{\mathrm{e}}$ personne et / ou à un temps du passé et engendrant dans la majorité des cas une structure complexe, semble en revanche être l'indice d'une écriture plus réfléchie convenant davantage au style narratif.

Mais cela dit, en Italie et en Gaule, tous les textes qui présentent, dans le sillage de dico, l'A.c.I. en antéposition, le présentent aussi en postposition : ainsi pour l'Italie, la Passio Caeciliae, la Passio Marcelli et Apuleii et la Vita Benedicti, et pour la Gaule, la Vita Martini, la Vita Walberti et la Vita Petri Venerabilis. Sept textes offrent uniquement des occurrences en postposition : en Italie, la Passio Agnetis, la Passio Agathae, la Passio Symphorosae et la Passio Viti (BHL 8711T), en Gaule, la Passio Saturnini, la Passio Benedictae et la Passio Iuliae. Donc du V ${ }^{\mathrm{e}}$ au VIII ${ }^{\mathrm{e}}$ ou IX ${ }^{\mathrm{e}}$ s. en Italie, du $\mathrm{IV}^{\mathrm{e}}$ au $\mathrm{XII}^{\mathrm{e}}$ s. en Gaule, l'A.c.I. reste bien vivant. Les trois seuls textes du corpus à éviter, du moins dans l'entourage de dico, l'A.c.I. au profit de quod p et de quia p sont respectivement la Passio Nazarii et Celsi et les Passio Pimenii et Passio Ansani et Maximae, trois textes écrits en Italie probablement du $\mathrm{VI}^{\mathrm{e}}$ au VIII ${ }^{\mathrm{e}}$ ou IX ${ }^{\mathrm{e}}$ s. ; la Passio Nazarii et Celsi offre même, on l'a vu, en l'absence de l'A.c.I., un exemple plausible de dico $+u t \mathrm{p}$ assertif. Donc, sur ce point, on voit en Italie un clivage se manifester entre les Vitae et les Passiones, les dernières attestant normalement un niveau de langue plus bas que les Vitae. En Gaule, la situation est différente dans la mesure où la subordination explicite est pour ainsi dire inexistante en ce qui concerne les déclaratives, Alors qu'en Italie, un tiers des séquences de déclaratives subordonnées (16 sur 48) se présentent sous forme d'une conjonctionnelle, ce type de subordination ne compte qu'une seule occurrence en Gaule (4,35\% des séquences, soit 1 sur 23). De plus, cette occurrence de quod p postposé a été repérée dans la Vita Petri Venerabilis ${ }^{44}$, un texte très tardif du XII ${ }^{\mathrm{e}} \mathrm{s}$.

Que l'A.c.I. ne soit pas seulement employé fréquemment mais aussi correctement est prouvé par le nombre très faible d'entorses à la norme classique ${ }^{45}$. D'une façon générale, l'usage des réfléchis y répond à la norme. Le sujet à l'accusatif est normalement correctement exprimé, même en cas de coréférence avec le sujet de dico, y compris à la $3^{\text {e }}$ personne, où l'on emploie couramment - en l'occurrence 15 fois - le réfléchi indirect $s e^{46} . M e$, te, se sujets d'une infinitive coréférentiels de l'agent de dicere, sont ainsi correctement exprimés en (21), (23) et $(26)^{47}$. Le pourcentage de coréférence entre le sujet du verbe à l'infinitif et celui du verbe dicere est d'ailleurs à peu près égal en cas d'antéposition $(35 \%=6 \mathrm{cas})^{48}$ et de postposition de l'A.c.I. $(32 \%=15 \text { cas })^{49}$, en l'occurrence environ un tiers de part et d'autre.

L'emploi de l'infinitif périphrastique est rare mais non inexistant pour l'expression de la postériorité, comme le montre :

(32) Grégoire, Vita Benedicti 2,38,32 (= 108) : Cum enim constet quia Paraclitus spiritus ex patre semper procedat et filio cur se filius recessurum dicit ut ille ueniat qui a filio numquam recedit. «Quar cant certe chose est, ke li espirs conforteres toz tens eist del pere et del filh, puor coi dist li filz

\footnotetext{
${ }^{44}$ Pour ces données statistiques, voir le tableau en annexe, à la fin de l'étude.

${ }^{45}$ Sur ce point, les propositions infinitives dépendant de dico dans le corpus hagiographique se distinguent très nettement de celles étudiées chez Frédégaire (Bodelot : 2014).

${ }^{46}$ D'autres formes plus rares de réfléchis indirects sont le pronom sibi (Passio Agnetis 1,6,6) et les possessifs suae (Sulpice Sévère, Vita Martini 1,16,5) et suum (Raoul de Cluny, Vita Petri Venerabilis 2,16,9).

${ }^{47}$ En cas d'antéposition de l'A.c.I, on trouve un seul exemple, à savoir Passio Marcelli et Apulei 1,8,30 : Marcellus ... sacrilegio pollui se dixit ... Littéralement : «? Marcellus (se) dit être sali d'un sacrilège ... », où la coalescence de se + dixit peut suggérer une évanescence du statut propositionnel (desentialization) de l'infinitive, suite au passage de se dans la régissante et à sa réinterprétation comme un réfléchi direct (Fruyt $2015: 25-28)$.

${ }^{48}$ Voir pour la $1^{\text {ère }}$ personne : Passio Marcelli et Apuleii 1,7,9; Sulpice Sévère, Vita Martini 1,27,7; pour la $3^{\text {e }}$ personne : Passio Marcelli et Apuleii 1,8,30 ; 1,24,2 ; Grégoire, Vita Benedicti 2,23,36 (= 90) ; 2,38,32 (= 108).

${ }_{49}$ Voir pour la $1^{\text {ère }}$ personne : Passio Marcelli et Apuleii 1,8,23; pour la $2^{\mathrm{e}}$ personne : Passio Caeciliae 1,20,10 ; Sulpice Sévère, Vita Martini 1,13,3; Passio Iuliae 1,7,2; pour la $3^{\mathrm{e}}$ personne : Passio Agnetis 1,6,7; Passio Agathae 1,13,6 ; Passio Marcelli et Apuleii 1,10,5 ; Grégoire, Vita Benedicti 2,7,23 ; 2,8,31 ; Sulpice Sévère, Vita Martini 1,14,5 ; 1,16,5 ; 1,17,5 ; 1,20,2 ; Passio Benedictae 1,6,3 ; Raoul de Cluny, Vita Petri Venerabilis $2,16,10$.
} 
soi en uoies aleir, par ke cil uenget ki unkes ne soi depart del filh ? $»^{50} /$ «Car comme il est établi que le Paraclet procède tout le temps du père et du fils, pourquoi le fils dit-il qu'il va s'éloigner pour que vienne celui qui ne s'éloigne jamais du fils?»

Dans un cas, l'infinitif présent sans auxiliaire modal est employé à la place d'une périphrase en -urum à l'intérieur d'un système hypothétique :

(33) Grégoire, Vita Benedicti 2,23,36 (=90): Eas quippe se communione priuare dixerat nisi mores suos et uerba corrigerent. «Quar il auoit dit ke il lur tolroit la communion, se eles n'amendassent lur constumes et lur paroles. »/ «De fait il avait dit qu'il les priverait de la communion si elles ne réformaient pas leur conduite et leur façon de s'exprimer. »

Notons que c'est le seul exemple dans lequel le traducteur ait renoncé à la subordination implicite savante en ancien français : le remplacement de l'A.c.I. par une proposition conjonctionnelle a permis de varier et de nuancer davantage l'expression des modes et des temps en rendant possible, en l'occurrence, l'emploi d'un conditionnel à la place du subjonctif, qui, avec sa valeur non marquée, pouvait passer pour la forme «normale » dans le système hypothétique de l'ancien français.

En latin, d'une façon générale, des incorrections concernant la syntaxe temporelle ou modale se manifestent plutôt dans les subordonnées dépendant de l'infinitive qu'au niveau de l'infinitive même ; il s'agit le plus souvent de manquements aux règles du discours indirect en concordance du passé :

(34) Passio Agathae 1,5,7: Sancta Agatha respondit dixisti deos tuos esse quos uera diuinitas demonstrat ${ }^{51}$ «Sainte Agathe répondit: 'Tu as dit que tes dieux sont ceux qu'on reconnait à leur vraie divinité.' »

(35) Grégoire, Vita Benedicti 2,15,32 (=80): Quamuis hoc Honoratus eius discipulus cuius mihi relatione conpertum est nequaquam ex ore illius audisse se perhibet sed quia hoc dixerit dictum sibi a fratribus fuisse testatur. ${ }^{52}$

En définitive, il semble donc qu'en ce qui concerne les complétives déclaratives de premier degré, dépendant directement du verbe dicere, les infractions à la concordance des temps affectent davantage la subordination explicite ${ }^{53}$ qu'implicite. $^{2}$

\section{Les interrogatives indirectes}

Comme pour les déclaratives indirectes, certaines tendances relevées dans les interrogatives indirectes chez Frédégaire ${ }^{54}$ se manifestent, à différents degrés, aussi dans les textes hagiographiques ; certaines sont absentes, d'autres s'y ajoutent, variations qui sont souvent fonction du style, du lieu et de l'époque de rédaction du texte en question.

\subsection{Les interrogatives indirectes totales / disjonctives}

\subsubsection{Représentation de -ne, nonne, num}

Les particules interrogatives -ne, nonne, num (et ses composés numquid, numquidnam) ne sont attestées dans le corpus hagiographique qu'en question directe. Ce qui frappe, c'est que num et ses équivalents composés, qui sont avec 23 occurrences les introducteurs de loin les plus fréquents de l'interrogation totale autonome dans le corpus, sont une marque exclusive des textes rédigés en Italie, num étant d'ailleurs avec une seule occurrence ${ }^{55}$ largement déficitaire par rapport à ses composés ${ }^{56}$. -

\footnotetext{
${ }^{50}$ En ancien français, la postériorité n'est pas exprimée ; « soi » calque une fois de plus le réfléchi indirect se, sujet de l'A.c.I. latin.

${ }^{51}$ Voir, par ex., aussi (28).

${ }^{52}$ Cet exemple de Grégoire, traduit en (6), atteste à la suite de quia un subjonctif parfait (dixerit) qui dépend d'un infinitif parfait passif (dictum fuisse).

${ }^{53}$ Pour quia p, on se rappellera ce qui a été dit par ex., à propos des exemples (10) à (14), des entorses à la concordance des temps si l'on opte pour une interprétation de l'assertive comme indirecte et non comme directe.

${ }^{54}$ Voir l'introduction au $\S 1$.

${ }^{55}$ Passio Victoris et Coronae 1,641,49.

${ }^{56}$ Ceux-ci sont surtout bien représentés chez Grégoire, qui connaît numquid et numquidnam, et dans la Passio Caeciliae et la Passio Viti, qui ne connaissent que numquid, ce dernier étant en plus attesté une seule fois dans la Passio Ansani et Maximae 1,5,12.
} 
$N e$ et nonne, quoique d'une façon générale très peu employés ${ }^{57}$, sont sporadiquement attestés en Gaule et en Italie. La supplantation de -ne par numquid est un phénomène qui s'observe en latin postclassique à partir de Sénèque (Bodelot 1990 : 31).

Tableau 6 :

Termes introducteurs de l'interrogation indirecte totale

\begin{tabular}{|l|c|}
\hline$-n e$ & 0 \\
\hline nonne & 0 \\
\hline num & 0 \\
\hline utrumne & 1 \\
\hline an & 6 \\
\hline si & 20 \\
\hline Total & 27 \\
\hline
\end{tabular}

La particule -ne a toutefois été repérée en subordonnée interrogative, accolée à utrum, pour introduire une interrogation totale simple :

(36) Grégoire, Vita Benedicti 2,38,22 (107): Sed quia ab infirmis potest mentibus dubitari utrumne ad exaudiendum ibi praesentes sint ubi constat quia in suis corporibus non sint ... « Mais par tant ke des enfermes penses puet estre doteit, se il la sont present per oir, u certe chose est ke il en lur cors ne sont mie ; / / "Mais parce que les esprits faibles peuvent se demander s'ils sont présents pour les exaucer là où l'on sait qu'ils ne sont pas présents corporellement, ... »

\subsubsection{Emplois et valeur de an}

$A n$ est la seule particule à intervenir presque autant de fois en interrogation indirecte qu'en interrogation directe, si n'introduisant, lui, que des interrogations indirectes.

Conformément à l'usage classique, an fonctionne d'abord dans le corpus hagiographique comme marqueur du second membre d'une interrogation disjonctive. Il n'y est jamais accompagné de utrum introduisant l'alternative. Dans deux interrogations difficilement identifiables comme directes ou indirectes, le premier membre est introduit par numquidnam ou reste sans marque :

(37) Grégoire, Vita Benedicti 2,21,19 (=86): Dic quaeso te numquidnam credendum est huic dei famulo semper prophetiae spiritum adesse potuisse an per interualla temporum eius mentem prophetiae spiritus inplebat. «Di ge te proi, doit l'om dunkes croire l'espir de prophetie a cest seriant de deu toz tens pooir estre present, u par entreuaz des tens sa pense emplissoit li espirs de prophetie ? / «Dis-moi, je t'en prie, s'il faut donc croire que l'esprit de prophétie pouvait être présent constamment auprès de ce serviteur de Dieu ou si c'était seulement par intervalles que l'esprit de prophétie éclairait sa pensée. »

(38) Grégoire, Vita Benedicti 2,30,16 (=96): Velim nosse haec tanta miracula uirtute semper orationis impetrabat an aliquando etiam solo uoluntatis exhibebat nutu. " Ge uoldroi conoistre, se il cez si grandes miracles prist toz tens par la uertut de l'orison, u se il les faisoit a la fie meismes par lo soul comant de sa uolenteit. $»^{58} /$ 《Je voudrais savoir si ces miracles tellement prodigieux, il les perpétrait toujours par la force de l'oraison ou s'il les effectuait parfois même par le seul acte de sa volonté. »

Dans deux autres interrogatives disjonctives, le premier volet est marqué par si:

(39) Passio Getulii, Cerealis et soc. 1,32,1: Licinius dixit si desideras uiuere an mori dicito mihi. «Licinius dit: 'Dis-moi si tu désires vivre ou mourir.' »

(40) Passio Victoris et Coronae 1,643,27: Et post triduum uenerunt milites uidere si mortuus esset an adhuc uiueret. «Et trois jours plus tard les soldats vinrent voir s'il était mort ou s'il vivait encore. »

\footnotetext{
${ }^{57}$ La particule -ne est attestée deux fois dans l'interrogation directe en Gaule, une fois dans l'interrogation indirecte en Italie, accolé à utrum (ex. 36); nonne est attesté quatre fois dans l'interrogation directe, dont deux fois en Italie et deux fois en Gaule.

${ }^{58}$ On aura remarqué qu'en (37), l'interrogative est transposée en ancien français sous forme directe, en (38), sous forme indirecte.
} 
Tableau 7 :

Termes introducteurs de l'interrogation indirecte disjonctive ${ }^{59}$

\begin{tabular}{|l|c|}
\hline X...an & $1 ?$ \\
\hline numquidnam ...an & $1 ?$ \\
\hline si...an & 2 \\
\hline Total & $2+2 ?$ \\
\hline
\end{tabular}

Comme introducteur d'une interrogation indirecte simple, non disjonctive, an est dans l'usage classique presque exclusivement confiné aux tours nescio an, haud scio an, incertum est an et dubito $a n^{60}$. Ces tours équivalent, par leur sens global, à des affirmations atténuées : «je ne sais pas si ne pas ; peut-être que »; an, qui a un sens dubitatif fondamentalement neutre, n'y est plus interprétable séparément (Bodelot 1987 : 77-80 ; 2003 : 255-258). Or, dans le corpus hagiographique, an est employé librement dans des interrogatives indirectes simples dépendant de verbes aussi variés que exploro, quaero, percunctor, sciscitor, uideo, fateor, dont la postposition occasionnelle exclut l'hypothèse d'un figement lexical.

Dans ces énoncés, an empiète ${ }^{61}$ sur l'emploi classique de -ne et de num et peut, à toutes les périodes considérées, aussi bien en Gaule qu'en Italie, être simplement transposé par «si » :

(41) Grégoire, Vita Benedicti 2,14,6 (= 78) : Cui dum protinus mandatum de monasterio fuisset ut ueniret ipse sicut perfidae mentis fuit an uir domini prophetiae spiritum haberet explorare conatus est. «A cui cant enhelement fut mandeit del monstier ke il uenist, il alsi com il astoit de senzfoge pense soi efforzat d'espier, se li hom del sanior auoit l'espir de prophetie.» / «Comme il avait immédiatement reçu ordre du monastère de venir, mécréant comme il était, il entreprit de vérifier si l'homme du Seigneur avait effectivement l'esprit de prophétie. »

(42) Sulpice Sévère, Vita Martini 1,5,5: Respondit christianum se esse. Quaerebat etiam ab eo an timeret. Tum uero constantissime profitebatur numquam se tam fuisse securum ... « Il répondit qu'il était chrétien. Il lui demandait aussi s'il avait peur. Il déclarait alors très fermement qu'il n'avait jamais été aussi serein. »

Sur le modèle des deux derniers passages cités, le subjonctif est de règle dans la subordonnée interrogative en concordance du passé ; le prédicat recteur est dans ce cas plus souvent postposé qu'antéposé (rapport 4 à 2).

Dans un cas, on a un énoncé complexe avec un enchâssement à plusieurs niveaux : an introduit une subordonnée de second degré imbriquée dans une volitive en $u t$ de premier degré :

(43) Sulpice Sévère, Vita Martini 1,18,1 : imperat ut an uerus esset hic nuntius fateretur. "il lui ordonna de lui dire si cette nouvelle était vraie. »

Assorti d'une concordance des temps classique, puisqu'à la suite du présent historique (imperat) celleci peut se faire, selon la norme, aussi bien d'après le sens que d'après la forme ${ }^{62}$, pareil enchâssement témoigne d'un niveau de langue soutenu; la correction grammaticale peut en même temps s'expliquer par la date de rédaction assez haute, en l'occurrence le $\mathrm{IV}^{\mathrm{e}} \mathrm{s}$., de la Vita en question.

Avec un verbe recteur figurant à un temps du présent, le subjonctif est attesté dans un seul exemple, à savoir :

\footnotetext{
${ }^{59}$ Le point d'interrogation marque les interrogatives qui pourraient se prêter aussi à une interprétation comme question directe.

${ }^{60}$ Voir par ex. A. Ernout \& F. Thomas $\left(1953^{2}: 316\right)$.

${ }^{61}$ Cet empiétement a amené les linguistes qui plaidaient primitivement pour un sens adversatif-négatif de an à y voir l'indice d'une évolution secondaire (voir par ex. R. Kühner \& C. Stegmann $1914^{2}$ : II, 2, 523 ; A. Ernout \& F. Thomas : $\left.1953^{2}: 317\right)$. Autrement dit, an aurait dès le latin postclassique commencé à perdre sa valeur originelle de «si ne pas », qu'il n'a probablement jamais possédée. Sur l'évolution peu plausible de an à partir de atne, voir G. Serbat (1981:3).

${ }^{62}$ Sur la double consecutio temporum possible, d'après la forme ou d'après le sens, avec un présent historique ou dit de narration, voir par ex. A. Ernout \& F.Thomas (1953² : 408-409), Ch. Touratier (1994: 608-609).
} 
(44) Passio Viti 1,14,2 (BHL 8713DT): tradamus inquit bestiis corpora illorum et uideamus an ueniat Christus illos liberare. "Livrons, dit-il, leurs corps aux bêtes sauvages et voyons si le Christ vient / viendra les délivrer. »

Dans les exemples présentant un indicatif en concordance du présent, on a dans tous les cas affaire à des interrogations disjonctives. Il s'agit d'abord des exemples (37) et (38), où, au vu du contexte, les interrogatives pourraient aussi se prêter à une interprétation directe, puis de l'exemple (39), qui, du fait de son introduction par si, semble être le seul exemple sûr d'une interrogation indirecte présentant dans le contexte de an un indicatif, et cela uniquement en concordance du présent ${ }^{63}$. Or cet indicatif est à verser, comme on le verra, sur le compte de si plutôt que de an.

\subsubsection{Répartition de l'indicatif et du subjonctif après si}

Le corpus hagiographique présente une palette nettement plus vaste et bigarrée d'emplois de $s i$ interrogatif que la Chronique originale de Frédégaire. Si l'on inclut les deux emplois de si en interrogation disjonctive attestés en (39) et (40), on compte 22 occurrences $^{64}$, dont 15 sont suivis d'un subjonctif, 7 d'un indicatif. $S i$ avec indicatif est confiné à quatre textes, tous rédigés en Italie probablement du début du $\mathrm{VI}^{\mathrm{e}}$ s. à l'orée du VIII ${ }^{\mathrm{e}}$, en l'occurrence, la Passio Agathae, la Vita Benedicti de Grégoire, la Passio Getulii, Cerealis et soc. (ex. 39)., la Passio Viti :

(45) Passio Agathae 1,12,1 : Quintianus dixit nunc uidebo si Christus tuus curabit te. "Quintien dit: 'À présent je verrai si ton Christ prendra soin de toi.' "

(46) Grégoire, Vita Benedicti 2,3,84 (=63): Sed quaeso respondeas si deserere fratres debuit quos semel suscepit. » Mais ge te proi ke tu respondes, se il deut laissier les freres, les queiz il prist une fie. ${ }^{65} /$ « Mais je te prie de me répondre s'il devait abandonner des frères qu'il avait adoptés une fois. »

(47) Passio Viti 1,4,3 (BHL 8711T): Nunc quia uideo te obstinata mente in tua uelle permanere sententia correptione interrogabo si te a coepto conamine potero reuocare. ${ }^{66}$ «À présent que je vois que tu veux à tout prix persister dans ton opinion, je vais voir par le châtiment si je pourrai te faire renoncer à ton dessein et entreprise. »

L'indicatif ne se rencontre qu'en concordance du présent ${ }^{67}$ : ce contexte est le plus accessible à la syntaxe modale de l'interrogation directe. Abstraction faite de l'interrogative double attestée en (39), si p est toujours postposé. Le verbe subordonné qui figure à l'indicatif présent, futur ou parfait est 5 fois sur $7(=71,43 \%)$ un verbe modal exprimant l'idée de « pouvoir, devoir ou vouloir / désirer ${ }^{68}$.

Le subjonctif, deux fois plus fréquent après $s i$ interrogatif que l'indicatif, se rencontre dans $2 / 3$ de ses emplois (10 sur 15) en concordance du passé :

(48) Grégoire, Vita Benedicti 2,28,12 (=94) : Cumque post paululum si id quod iusserat datum esset inquireret ... «Et quant il apres un petit demandoit, se ce fust doneit ke il comandat, ...»/ «Et comme un peu plus tard il lui demandait s'il avait bien donné ce qu'on lui avait commandé de donner, ... »

(49) Vita Madelbertae 2,4,4: Quae sciscitata a matre si carnali se uellet sociare coniugio ac paternas percipere traditiones more solito illa cum lacrimis prae gaudio matri cogenti responsum diuinitus

\footnotetext{
${ }^{63}$ Voir en effet, à titre de comparaison, le subjonctif attesté en concordance du passé dans l'interrogation indirecte double marquée par si...an en (40).

${ }^{64}$ Parmi les verbes introducteurs de ces subordonnées en si, le plus fréquent est uideo; sont ensuite bien représentés les uerba interrogandi et inuestigandi, comme interrogo, quaero, inquiro, requiro, sciscito, exploro, mais aussi les uerba declarandi, comme dico, respondeo, indico, innotesco au sens de «faire connaitre », tous employés dans un contexte qui ne laisse subsister aucun doute sur l'orientation interrogative de si p.

${ }^{65}$ Voir aussi Grégoire, Vita Benedicti 2,32,38 (=100): Sed quaeso te indices si sancti uiri omnia quae uolunt possunt et cuncta inpetrant quae desiderant obtinere. «Mais je te prie de m'indiquer si les saints peuvent tout ce qu'ils veulent et s'ils obtiennent tout ce dont ils désirent disposer. »

${ }^{66}$ Voir aussi Passio Viti 1,15,4 (= BHL 8711T) ; 1,15,3 (= BHL 8712T), tous les deux cités en (55).

${ }^{67}$ Cette particularité peut s'expliquer par le fait qu'après un verbe au passé, notamment s'il figure à la $3^{\text {e }}$ personne, le subjonctif de l'interrogation indirecte est assez proche d'un subjonctif de style indirect. Voir à ce propos C. Bodelot (2003 : 325), qui renvoie à J.-L. Moralejo (1996).

${ }^{68}$ Le verbe subordonné à l'indicatif n'est pas modal dans Passio Agathae 1,12,1 (= ex. 45) ; Passio Viti 1,15,3 $(=$ BHL $8712 \mathrm{~T})\left(=\right.$ le $2^{\text {nd }}$ ex. cité en 55$)$.
} 
attulit dicens ... « Interrogée par la mère si elle voulait se lier par une union charnelle et suivre les enseignements de ses pères selon la pratique habituelle, elle donna, pleurant de joie, à la mère qui l'y poussait une réponse inspirée par Dieu en disant ... »

(50) Raoul de Cluny, Vita Petri Venerabilis 2,15,15 : Explorabat etiam si confessus fuisset si esset absque poena si communionem suscepisset. ${ }^{69}$ «Il s'informait aussi s'il s'était confessé, s'il avait eu l'absolution, s'il avait reçu la communion. »

dans $1 / 3$ de ses emplois ( 5 sur 15 ) en concordance du présent :

(51) Grégoire, Vita Benedicti 2,8,89 (=72): Quaeso te post haec ad quae loca uir sanctus migrauerit uel si aliquas in eis uirtutes ostenderit innotesce. » Ge te proi ke tu faces conoistre apres cez choses, a queiz lius s'en alat li sainz hom, u se il demostrat en iceaz alcunes uertuz. » / « Je t'en prie, apprendsmoi à la suite de ce récit en quels lieux ce saint homme s'est retiré et s'il y a fait des miracles. »

(52) Passio Viti 1,15,3 (BHL 8713DT) : Volo inquit uidere si deus tuus eruat te de manibus meis. ${ }^{70}$ « Je veux voir, dit-il, si ton Dieu t'arrache / arrachera de mes mains. »

(53) Passio Ansani et Maximae 1,8,19: Sed si uultis ut adorem deos uestros inquiramus quorum sint isti ipsi imagines et uideamus si his merito honorem exhibere debeamus. "Mais si vous voulez que j'adore vos dieux, faisons une enquête sur ceux dont nous voyons les portraits ici et voyons s'ils méritent vraiment que nous les vénérions. »

Tout comme si p à l'indicatif, si $\mathrm{p}$ au subjonctif est dans la grande majorité des cas (13 sur $15=$ $86,67 \%)$ postposé $^{71}$. À l'opposé de ce qu'on a constaté pour les verbes subordonnés à l'indicatif, les verbes au subjonctif ne sont des auxiliaires modaux que dans $4^{72}$ cas sur $15(=26,67 \%)$. Autre différence significative : alors qu'un seul des verbes subordonnés à l'indicatif ${ }^{73}$ exprimait un rapport d'antériorité, ce rapport est exprimé dans la moitié des cas $\left(8^{74}\right.$ sur 15$)$ au subjonctif.

On en conclura que l'emploi de l'indicatif en interrogation indirecte introduite par si est nettement plus contraint que celui du subjonctif : il est confiné dans les textes hagiographiques rédigés en Italie ${ }^{75}$, n'est attesté qu'en concordance du présent et n'est guère employé pour exprimer un rapport temporel d'antériorité ; en plus, le verbe à l'indicatif est dans la grande majorité des cas un auxiliaire modal.

$\mathrm{Si}+$ subjonctif se rencontre au contraire aussi bien dans l'hagiographie gauloise qu'italienne ; même s'il se rencontre de préférence dans un contexte du passé, il n'est pas incompatible avec une concordance au présent; le verbe au subjonctif peut exprimer un rapport temporel de simultanéité d'antériorité ou de postériorité, même si ce dernier n'est jamais signifié par une forme périphrastique en -urus mais par le subjonctif présent ou imparfait, surtout d'un verbe modal.

Mais ici encore il faut être prudent et ne pas ériger en règles de simples tendances. À quel point la distribution des modes et l'expression de la postériorité sont soumises à fluctuation ressort notamment de deux énoncés de la Passio Viti, qui ont été transmis sous deux formes différentes :

(54) Passio Viti 1,14,2 (BHL 8711T): Tunc Diocletianus furore succensus iussit arenarium praeparari dicens bestiis ferocissimis tradam eos ut uideam si Christus eorum possit liberare eos de manibus meis. "Alors Dioclétien furieux ordonna de préparer l'arène et dit: 'Je les livrerai aux bêtes sauvages pour voir si leur Christ peut / pourra les délivrer de mes mains.' »

Vs. Passio Viti 1,14,1 (BHL 8712T) : Turbatus imperator dixit ad bestias tradam animas eorum et uidebo si Christus liberet eos de manibus meis. "Bouleversé, l'empereur dit: 'Je livrerai leurs vies aux bêtes sauvages et je verrai si le Christ les délivre / délivrera de mes mains.' »

${ }^{69}$ Voir aussi Grégoire, Vita Benedicti 2,21,25 ; Passio Victoris et Coronae 1,643,27, cité en (40); Raoul de Cluny, Vita Petri Venerabilis 2,9,7;2,10,18;2,15,24.

${ }^{70}$ Voir aussi Passio Viti 1,14,2 (= BHL 8711T) ; 1,14,1 (= BHL 8712T), tous les deux cités en (54).

${ }^{71} \mathrm{Si}$ p est antéposé en (48) et (51).

${ }^{72}$ Il s'agit de Grégoire, Vita Benedicti 2,21,25 ; Passio Viti 1,14,2 (BHL 8711T) (= ex. 54) ; Passio Ansani et Maximae 1,8,19 (= ex. 53) ; Vita Madelbertae 2,4,4 (= ex. 49).

${ }^{73}$ Il s'agit du modal debuit en (46).

74 Il s'agit de Grégoire, Vita Benedicti 2,8,89 (=ex. 51) ; 2,28,12 (= ex. 48); Raoul de Cluny, Vita Petri Venerabilis $2,9,7 ; 2,10,18 ; 2,15,15$ (bis) (= ex. 50) ; 2,15,24; Passio Victoris et Coronae 1,643,27 (= ex. 40).

${ }^{75}$ On rapprochera ce point de ce que nous avons dit plus haut de l'attestation quasi exclusive des déclaratives conjonctionnelles en Italie. Donc il semble que l'hagiographie italienne soit, d'une façon générale, plus accessible aux vulgarismes morphosyntaxiques que l'hagiographie gauloise. 
(55) Passio Viti 1,15,4 (BHL 8711T) : Tunc prae nimio furore se ipsum non capiens imperator iussit ministris parare clibanum et in eo resolui plumbum et resinam et picem. Feceruntque ministri sicut eis praeceperat imperator et deposuerunt in eum beatum athletam Christi Vitum. Dum deponeretur dixit imperator ecce modo uideo si deus tuus poterit liberare te de manibus meis. "Alors ne se contenant plus, en proie comme il était à une terrible crise de rage, l'empereur ordonna à ses serviteurs de préparer le four et d'y dissoudre du plomb, de la résine et de la poix. Les serviteurs exécutèrent les ordres de l'empereur et y déposèrent le bienheureux athlète Vitus. Alors qu'on l'y déposait l'empereur dit: 'Voilà que je vais vite voir si ton Dieu pourra te délivrer de mes mains.' »

Vs. Passio Viti 1,15,3 (BHL 8712T): Tunc imperator furore repletus iussit ministris clibanum preparare et solui plumbum et resinam et picem. Et fecerunt ministri sicut preceperat eis. Diocletianus dixit ecce modo uidebo si deus tuus liberat te de his poenis. "Alors l'empereur dans un accès de rage ordonna à ses serviteurs de préparer le four, d'y dissoudre du plomb, de la résine et de la poix. Les serviteurs exécutèrent les ordres de l'empereur. Dioclétien dit: 'Voilà que je verrai vite si ton Dieu te délivre / délivrera de ces supplices.' »

Que l'on recoure à un verbe simple ou à un verbe modifié par un auxiliaire, subjonctif présent et indicatif présent ou futur simple semblent avoir été en Italie, dans la seconde moitié du $1^{\text {er }}$ millénaire, autant de candidats possibles à l'expression de la postériorité en concordance du présent dans l'interrogation indirecte introduite par si.

En conclusion, on retiendra que les textes hagiographiques semblent confirmer ce que nous avons eu l'occasion de constater à propos d'autres textes postclassiques ou tardifs (Bodelot 1999: 219-220), à savoir que si crée plus que n'importe quel autre interrogatif un contexte favorable à l'emploi de l'indicatif. C'est que par l'histoire de son emploi ${ }^{76}$, en l'occurrence sa filiation de $s i$ hypothétique, il était, même sans subjonctif, facilement reconnaissable comme subordonnant. Attesté avec des verbes recteurs exprimant ou non une attitude de doute chez le sujet modal ou le locuteur, cet emploi de l'indicatif après si a dû jouer un rôle important dans l'extension progressive de ce mode en subordonnée interrogative lors du passage du latin aux langues romanes (Bodelot 2003 : 324-325; $2013: 372)$.

\subsection{Les interrogatives indirectes partielles}

Pour ce qui est de l'expression de la cause et de la manière dans l'interrogation indirecte, on relève certaines différences notables par rapport à la Chronique originale de Frédégaire.

\subsubsection{L'expression de la manière}

Tableau 8 :

Termes introducteurs de l'interrogation indirecte de manière ${ }^{77}$

\begin{tabular}{|l|c|}
\hline quo ordine & 2 \\
\hline quemadmodum & 1 \\
\hline ut & 1 \\
\hline qualiter & $8+2 ?$ \\
\hline quomodo & $14+2 ?$ \\
\hline Total & $26+4 ?$ \\
\hline
\end{tabular}

Quemadmodum ${ }^{78}$ et quo ordine sont communs aux deux corpus. Tandis que les deux adverbes interrogatifs sont suivis dans l'interrogation indirecte chez Frédégaire tantôt de l'indicatif tantôt du subjonctif, quemadmodum est suivi dans le corpus hagiographique, dans le seul exemple sûr d'interrogation indirecte où il soit attesté ${ }^{79}$, d'un subjonctif de valeur délibérative, quo ordine étant suivi, lui, une fois du subjonctif, une fois de l'indicatif :

\footnotetext{
${ }^{76}$ Sur le développement de si interrogatif à partir de si conditionnel ou hypothétique, lui aussi subordonnant, voir C. Bodelot (2003 : 258-263; 2013), avec références bibliographiques.

${ }^{77}$ Le point d'interrogation marque les emplois des termes qui pourraient aussi se prêter à une interprétation conjonctionnelle au sens de «que».

${ }^{78}$ En l'occurrence, graphié quemammodum; voir l'ex. (56).

${ }^{79}$ Dans Passio Victoris et Coronae 1,642,23, l'interrogative en quemadmodum à l'indicatif peut à la suite de Dic mihi être interprétée comme directe.
} 
(56) Passio Marcelli et Apuleii 1,6,12: Tunc praecepit eum praeses teneri et retrudi in carcerem donec uotiuos dies transactos excogitaret quemammodum eum perderet. "Alors le gouverneur le fit arrêter et emprisonner jusqu'à ce que, les jours de vœux terminés, il eût une idée comment il pouvait le perdre. »

(57) Passio Caeciliae 1,17,34: Almachius dixit et ego quare non aduerto quo ordine ista prosequaris. «Almachius dit : 'Et pourquoi est-ce que moi, je ne me rends pas compte de quelle façon tu parviens à cette constatation'?»

(58) Grégoire, Vita Benedicti 2,22,34 (= 88) : Doceri uelim quo fieri ordine potuit ut longe iret responsum dormientibus diceret quod ipsi per uisionem audirent et recognoscerent. " Ge uoldroie ke l'om moi enseniast, par queil ordene ce pout estre fait, ke il iroit lonz, ke il az dormanz diroit respeus, ke cil l'oroient par uision et reconistroient ? / « Je voudrais qu'on m'apprenne de quelle façon il a pu se faire qu'il s'en allât au loin, transmît à des personnes endormies une consigne qu'ils pouvaient entendre et reconnaître clairement en songe. »

Outre le fait qu'en (58), à la suite de Doceri uelim, il n'est pas impossible de voir dans l'interrogative une question directe, l'indicatif pourrait aussi y être calqué sur le tour quo ordine fieri potest ut, attesté ailleurs chez Grégoire ${ }^{80}$.

Alors que quo pacto est absent du corpus hagiographique, on y repère comme autres adverbes de manière introduisant une interrogation indirecte ut, qualiter et quomodo, qui font défaut chez Frédégaire.

$V t$, qui était rare et désuet dans l'interrogation directe mais usuel dans l'interrogation indirecte du latin préclassique au latin postclassique (Bodelot 1990 : 16-17, 29, 38-39, 44), est attesté une seule fois dans le texte le plus ancien du corpus, correctement suivi du subjonctif :

(59) Sulpice Sévère, Vita Martini 1,1,7 : Igitur sancti Martini uitam scribere exordiar ut se uel ante episcopatum uel in episcopatu gesserit ... « Je commencerai donc à écrire la vie de saint Martin, < et à dire > comment il s'est comporté avant ou pendant son épiscopat ... »

Qualiter, d'un emploi rare en latin classique mais courant en latin tardif (Hofmann-Szantyr 1972²: 459), est, dans le corpus, confiné à l'emploi subordonnant ${ }^{81}$. Dans l'emploi interrogatif, il est avec 10 occurrences le concurrent le plus puissant de quomodo, qui compte 16 occurrences, dont 7 dans un seul et même énoncé chez Raoul de Cluny :

(60) Raoul de Cluny, Vita Petri Venerabilis 2,7, 63-66: In primis dicit quam deuote eum susceperit quomodo infirmatus quomodo confessus sit quomodo id quod sibi uerecundiam inferebat reticuit quomodo raptus quomodo accusatus quomodo excusatus quomodo superueniens mater misericordiae eum de manibus malignorum eripuit uitae reddidit et ut peccatum quod tacuerat confiteretur admonuit. " Il dit avant tout avec quel dévouement il le recueillit, comment il fut malade, comment il confessa ses péchés, comment il garda le silence sur celui qui lui inspirait de la honte, comment il fut traîné en justice, comment il fut inculpé, comment il fut disculpé, comment la mère miséricordieuse, venant à son secours, l'arracha aux mains des malins, le rendit à la vie et lui conseilla de confesser le péché qu'il avait tu. »

Un seul texte, à savoir la Passio Pimenii, présente l'un et l'autre terme :

(61) Passio Pimenii 1,3,28: Tu uero qui Romanae sedis illustris es ac semper diis seruisti a quibus ego humilis exaltatus sum quomodo seductus es ignoro. "Quant à toi qui es d'illustre origine romaine et as toujours servi les dieux, qui, humble comme j’étais, m’ont élevé en dignité, j'ignore comment tu as été détourné du droit chemin. »

\footnotetext{
${ }^{80}$ Voir supra l'exemple (12). Pour ce qui est de l'ancien français, on remarque que quoniam n'y est pas traduit ; comme l'intonation de cet énoncé nous échappe suite à une ponctuation incertaine, il est difficile de savoir si la proposition qui suit doit être directement rattachée à «congeteir» ou non. Autrement dit, la proposition interrogative «par queil ordene puet estre fait...» semble pouvoir être interprétée dans la transposition en ancien français aussi bien comme directe que comme indirecte.

${ }^{81}$ Comme relatif, interrogatif et peut-être conjonctif ; voir Passio Viti 1,6,4 (BHL 8711T), où il introduit une comparative de manière
} 
(62) Passio Pimenii 1,6,2-4: ... non absurdum credimus si qualiter omnipotens deus post transitum beati Pigmenii presbiteri et martiris uindictam in crudeli et impio Iuliano sit operatus uel qualiter faciente domino prout in gestis inuenimus crudelissimus regnum cum uita perdidit breuiter fideliterque notitiae fidelium enarremus. «... il ne nous paraît pas déplacé de transmettre brièvement et honnêtement à la connaissance des fidèles de quelle façon le Dieu tout-puissant, après le trépas du bienheureux prêtre et martyr Pigmène, a châtié le cruel et impie Julien et de quelle façon, à l'instigation du Seigneur, d'après ce que nous avons lu dans ses actes, ce personnage très cruel a perdu la souveraineté royale en même temps que la vie. »

Par ailleurs, quomodo semble avoir été répandu plus largement et plus tôt en hagiographie italienne que gauloise, où il n'est attesté que chez Raoul de Cluny au XII $\mathrm{e}$ s. Tout comme quomodo, qualiter est présent dans le corpus italien dès le $\mathrm{VI}^{\mathrm{e}}$ s.; en Gaule, qualiter est attesté dans des textes du $\mathrm{IX}^{\mathrm{e}} \mathrm{s}$. si bien qu'on a l'impression qu'il y a été relayé ultérieurement par quomodo.

Qualiter n'est suivi de l'indicatif (perdidit) que dans l'exemple $(62)^{82}$ de la Passio Pimenii ; il est en dépendance d'un même verbe recteur (enarremus) coordonné à une autre interrogation indirecte en qualiter, qui figure, elle, au subjonctif (sit operatus) sans qu'il soit possible de détecter une différence de sens entre les deux modes. La même Passio Pimenii présente encore l'indicatif en (61), dans une interrogative en quomodo. Or avec un verbe recteur comme ignoro, qui exprime sans ambages l'absence de savoir, on aurait pu s'attendre à l'emploi du subjonctif. On y verra les indices d'un emploi peu conséquent des modes, qui s'inscrit dans une syntaxe de subordination relâchée.

Une alternance des modes s'est observée ensuite avec quomodo dans l'exemple (60). La fluctuation semble ici s'expliquer par la longueur et la complexité de l'énoncé, l'indicatif relayant le subjonctif au fur et à mesure qu'on s'éloigne du verbe recteur dicit. Dans d'autres interrogatives subordonnées plus brèves et de structure transparente, Raoul emploie à la suite de quomodo le subjonctif ${ }^{83}$.

Un exemple d'interprétation plus délicate parce qu'attestant l'indicatif en concordance du passé est :

(63) Passio Nazarii et Celsi 1,6,5: Audiens haec Anolinus qui eum iam ex urbe Mediolanensi expulerat quod haec faceret beatus Nazarius repletus indignatione dixit militibus suis quomodo effugit manus Neronis homo iste. «À cette nouvelle, Anulinus, qui l'avait déjà chassé de la ville de Milan, se scandalisant de la conduite du bienheureux Nazaire, raconta à ses soldats comment cet homme s'était échappé des mains de Néron. »

Puisqu'ailleurs dans le même texte on trouve employé après quomodo introduisant une interrogation indirecte le subjonctif même pour un auxiliaire modal au présent ${ }^{84}$, on peut se demander si l'on n'a pas affaire ici à un quomodo grammaticalisé ${ }^{85}$ qui, à la suite d'un uerbum dicendi, s'est vidé de son sens percontatif de manière, et est passé du statut d'adverbe à celui de conjonction de subordination ${ }^{86}$, introduisant au sens de «que » une complétive assertive ${ }^{87}$.

Le même usage conjonctionnel de quomodo, cette fois suivi du subjonctif, est peut-être attesté un demi-millénaire plus tard en Gaule chez Raoul de Cluny, à condition que l'on donne à mirari le sens de «s'étonner (que) » et non de « se demander avec étonnement (comment)» :

\footnotetext{
${ }^{82}$ Un deuxième énoncé présentant une interrogation indirecte introduite par qualiter (coordonné à qua de causa), à savoir Passio Pimenii 1,2,19, fait l'économie du verbe subordonné ; voir l'ex. (71), cité infra.

${ }^{83}$ Voir Raoul de Cluny, Vita Petri Venerabilis 2,2,2 ; 2,2,37 ; 2,4,15 ; 2,15,13.

${ }^{84}$ Voir Passio Nazarii et Celsi 1,1,17 : Ipse mihi ostendat quae sunt iusta et uiam suam quomodo oporteat me ingredi et egredi coram ipso. "Que lui-même me montre ce qui est juste et de quelle façon il faut que j'emprunte son chemin et le quitte sous son regard. »

${ }^{85}$ Sur l'emploi de quomodo et aussi de qualiter (cf. infra) dans des contextes ambigus admettant l'une ou l'autre interprétation, voir aussi P. Stotz (1998 : 400-401).

86 Selon J. Herman (1957 : 375), quomodo conjonctionnel est justement issu de quomodo employé dans des interrogations indirectes qui ne dépendent pas de verbes exprimant par eux-mêmes une idée d'interrogation. Tout en concédant que cet usage a pu se développer sans influence étrangère, J. Pirson (1908: 71) constate que cet emploi s'est trouvé d'abord chez des écrivains qui s'inspiraient d'ouvrages grecs. G. Mayen (1889 : 43-45) considère, d'une façon plus radicale, cet usage comme un pur grécisme. Voir, à ce propos, notre étude de quomodo chez Lucifer de Cagliari (Bodelot 2012).

${ }^{87}$ Sur l'emploi de quomodo au sens de «que » suivi de l'indicatif à la suite de audio chez sainte Brigitte de Suède, voir E. Karlsen (2001 : 39)
} 
(64) Raoul de Cluny, Vita Petri Venerabilis 2,4,15: Quod reges terrae audientes mirati sunt quomodo monachum suum in sede positum relinqueret et extraneum exaltaret ... « À cette nouvelle, les rois du monde se demandèrent avec étonnement comment il pouvait laisser son moine assis sur son siège et élever en dignité un étranger ...»

Il est possible qu'un processus analogue de grammaticalisation soit à l'œuvre avec qualiter, suivi du subjonctif, dans :

(65) Passio Getulii, Cerealis et soc. 1,111,1 : Et iratus ualde citius cucurrit nuntiauit haec Adriano qualiter Cerialis uicarius factum se esse christianum fateretur. " Emporté par la colère, il courut vite annoncer à Hadrien de quelle façon le vicaire Cerealis proclamait être devenu chrétien. »

(66) Passio Benedictae 1,9,8: Denique studebat multopere minister daemonis et totius fomes criminis qualiter ipsius pretiosae uirginis animam ad caeli palatium properantem ad inferni claustra mitteret et Christi sanguine redemptam denuo daemonicae iugo seruitus inclinaret. «Enfin le serviteur du démon, qui avait suscité toute l'accusation, s'appliquait vaillamment à renvoyer dans l'enceinte de l'enfer l'âme de cette excellente jeune fille qui se hâtait de rejoindre le palais du ciel et, alors qu'elle avait été rachetée par le sang du Christ, à la faire tomber de nouveau sous le joug de l'esclavage du démon. »

Dans (65), qualiter pourrait, en dépendance de nuntiauit, commuter sans différence de sens notable avec quod ou une proposition infinitive, dans (66), en dépendance de studebat, avec ut introduisant une complétive volitive ${ }^{88}$.

De tous ces marqueurs de manière, c'est quomodo qui a le mieux résisté en roman ${ }^{89}$, qualiter n'ayant pas été en aval aussi prospère que qualis. Suite au processus de grammaticalisation amorcé en latin, les dérivés romans de quomodo continuent à introduire des complétives assertives après les verbes de déclaration et de perception, notamment dans le vieil espagnol et le vieux portugais ${ }^{90}$.

\subsubsection{L'expression de la cause}

À l'instar de l'expression de la manière, les interrogations indirectes portant sur la cause sont dans le corpus hagiographique pourvus de marqueurs variés.

Tableau 9 :

Termes introducteurs de l'interrogation indirecte de cause

\begin{tabular}{|l|l|}
\hline cur & 1 \\
\hline qua de causa & 2 \\
\hline quare & 1 \\
\hline Total & 4 \\
\hline
\end{tabular}

L'emploi indirect de cur n'est pas le même que chez Frédégaire puisqu'à côté d'énoncés du type de :

(67) Sulpice Sévère, Vita Martini 1,22,3 : Testabantur etiam aliqui ex fratribus audisse se daemonem proteruis Martinum uocibus increpantem cur intra monasterium aliquos ex fratribus qui olim baptismum diuersis erroribus perdidissent conuersos postea recepisset ... « Certains des frères attestaient même qu'ils avaient entendu le démon blâmer impudemment Martin parce qu'il avait / d'avoir recueilli dans le monastère, après leur conversion, quelques frères qui avaient perdu la grâce du baptême suite à divers égarements ... »

(68) Sulpice Sévère, Vita Martini 1,23,4: Ille ei iram domini et praesentes plagas cur sancto non crederet comminari. "Il le menaçait de la colère du Seigneur et de coups immédiats parce qu'il ne croyait pas un saint. »

\footnotetext{
${ }^{88}$ Sur qualiter évoluant tout comme quomodo vers le sens de quod, quia et quoniam, voir J. Pirson (1908 : 71$)$. Sur la valeur factuelle faible de qualiter conjonctionnel s'employant au $\mathrm{X}^{\mathrm{e}}$ s. dans le Chronicon Salernitanum à la suite d'un uerbum sentiendi, voir P. Greco (2012 : 60-61).

89 Voir dans DHELL notre contribution sur quomodo (Bodelot: 2015), notamment le $\S 7$ sur la filiation du lexème dans les langues romanes (avec indications bibliographiques). Pour une étude de fr. «comme»et « comment » s'inscrivant dans une perspective diachronique large, voir V. Wielemans (2005).

${ }^{90}$ Voir à ce propos encore J. Herman (1957 : 375-376), avec des exemples illustrant dans les deux langues l'équivalence entre como et que.
} 
où il exprime la cause à la suite d'un verbe signifiant blâme ou menace, ces textes offrent aussi une interrogation indirecte introduite par cur purement percontatif :

(69) Passio Agathae 1,4,21: Dic tamen antequam ad tormenta uenias cur deorum sancta contemnas. « Dis-moi cependant avant que tu subisses la torture pourquoi tu méprises les lois de nos dieux. »

La même valeur percontative est véhiculée dans le corpus par le composé qua de causa. Ne comptant que deux occurrences, il n'est pourtant pas propre à une époque ou à une région : il est attesté une fois, au XII ${ }^{\mathrm{e}}$ s., chez Raoul de Cluny en Gaule, une autre fois beaucoup plus tôt, peut-être au VI ${ }^{\mathrm{e}}$ s., dans la Passio Pimenii en Italie, en coordination avec qualiter, sans verbe subordonné exprimé :

(70) Raoul de Cluny, Vita Petri Venerabilis 2,10,7 : Tunc fratres ad eum concurrentes quaerebant ab eo qua de causa sic clamaret. "Alors accourant vers lui, les frères lui demandaient pourquoi il criait de la sorte. »

(71) Passio Pimenii 1,2,19: ... Pygmenius Persidam et beatus Donatus Aritium Tusciae oppidum petiit. Qualiter autem quaue de causa notitiae fidelium in sequentibus fideliter commendare curemus. «... Pigmène gagna la Perse et le bienheureux Donat la ville d'Aritium en Etrurie. Or de quelle façon et pour quelle raison < ils prenaient cette direction>, empressons-nous, dans ce qui suit, de le transmettre avec honnêteté à la connaissance des fidèles. »

Avec 23 occurrences, le composé quare, accompagné ou non de non, est dans le corpus hagiographique l'interrogatif de cause de loin le plus fréquent en question directe, que ce soit en question informative ou rhétorique. Il n'est en revanche affecté qu'une seule fois à l'introduction d'une subordonnée, en Italie peut-être dès le $V^{e} s$. :

(72) Passio Caeciliae 1,17,34: ... et ista attendentes miror quare non erubescimus militum Christi uictorias silentio tegere et non ad laudem imperatoris caelestis qualiter pugnauerint contra hostes et uicerint scedulis saltem uilibus tradere et ad incitandos animos bellatorum diligentius explicare. «... et je me demande avec étonnement pourquoi, en voyant cela, nous n'avons pas honte / je m'étonne ... que ... nous n'ayons pas honte / je suis frappé d'étonnement ... parce que nous n'avons pas honte de taire les victoires des soldats du Christ, de ne pas transmettre, à la gloire du céleste souverain, ne fût-ce que sur des feuillets de peu de valeur, de quelle façon ils ont victorieusement combattu contre les ennemis et de ne pas le développer avec plus de zèle pour stimuler le courage des guerriers. »

Bien que cet exemple ne réponde pas de tous les points de vue au schéma phrastique traditionnel (verbe exprimant un sentiment négatif ou verbe de punition ou de vengeance + interrogation indirecte) dont on dérive normalement le cur ou le quare causal ${ }^{91}$, il a pu contribuer de deux façons distinctes mais complémentaires à cette même filiation. Ou bien quare, dont l'interprétation comme adverbe interrogatif (au sens de «pourquoi ») reste ici possible, a été perçu, dans le sillage de miror transitif, comme l'équivalent de quod et de quia complétifs (au sens de «que »); puis, par analogie avec quod et quia ${ }^{92}$, suite à un glissement imperceptible du contenu du sentiment à son motif, quare a pu être compris lui-même comme une conjonction de cause (au sens de "parce que ») après un verbe de sentiment qui ne requérait pas forcément de second actant. Ou deuxième filiation, plus immédiate, possible : dans un même contexte originellement interrogatif, quare a pu être interprété directement, sans le moindre détour par quod ou quia complétifs, comme une conjonction causale à la suite de miror, perçu comme intransitif, au sens de «être frappé d'étonnement (parce que)».

Quoi qu'il en soit, à supposer que la réanalyse d'une même structure en contexte «critique » ait pu conduire, par paliers, de l'emploi de quare comme adverbe interrogatif indirect à son emploi comme conjonction de subordination causale, on peut rendre compte de différents degrés d'hypotaxe, dont

\footnotetext{
${ }^{91}$ J. Herman (1957) parle d'une « ressemblance indéniable » entre les deux évolutions, celle de cur et de quare, tout en concédant que « le problème paraît être ... plus compliqué » pour quare (ibid. : 372), parce que «quare étant non seulement plus populaire que cur, mais ayant aussi, d'emblée, des emplois plus variés, des glissements occasionnels ont pu se produire dans la parole vers le sens de conjonction causale ... » (ibid. : 373).

${ }^{92}$ Pour le rapprochement avec quod et quia, voir J. B. Hofmann \& A. Szantyr $\left(1972^{2}: 541\right)$, qui citent à ce propos un exemple comparable avec miror chez Mart. 4,39,10: argentum tamen inter omne miror quare non habeas ... purum.
} 
« car » continue à témoigner à l'intérieur du français, et cela jusqu'à l'époque moderne, où son statut supposé de coordonnant est souvent loin d'être clair ${ }^{93}$.

\section{Conclusion}

Pour les complétives assertives, on retiendra que l'A.c.I. reste dans le corpus la construction la plus répandue aussi bien en Gaule qu'en Italie. L'infinitive résiste donc bien - plus qu'on ne l'affirme parfois - à la concurrence des subordonnées introduites par quod, quia, ut et, dans une moindre mesure, quomodo. Même si le latin produit en Italie semble à maint égard plus novateur et ouvert aux constructions vernaculaires que le latin gaulois, le maintien d'une concordance des temps somme toute classique confirme le caractère généralement conservateur de ce corpus hagiographique. De plus, en cas de postposition au verbe dicere, l'emploi de l'A.c.I. est, à la différence de celui des complétives conjonctionnelles - susceptibles de le concurrencer en cette position -, beaucoup plus libre et pour ainsi dire exempt de contraintes ${ }^{94}$.

Vu l'écrasante prépondérance et la liberté d'emploi de la subordination implicite, il ne semble donc guère possible de parler, pour l'assertion indirecte, d'une réelle rivalité exercée par la subordination explicite. Si concurrence et rivalité il y a, elle provient de la parataxe. On voit en effet très souvent une instance de discours direct se substituer à une complétive assertive attendue - ou au moins tout aussi acceptable - à la suite du verbe dicere.

La même remarque peut se faire en ce qui concerne les interrogatives indirectes, où le remplacement du mode subjonctif par l'indicatif dans les véritables subordonnées ne se fait que lentement et surtout dans les cas où le subordonnant offre une compensation formelle au marquage par le mode. Certains traits de la syntaxe modale ne se laissent ici encore expliquer que par référence à la question directe, si bien qu'on hésite, en concordance du présent, parfois entre l'une et l'autre interprétation. Or la préférence pour le discours direct a moins à faire, comme on le sait, avec l'état de la langue qu'avec le niveau de la langue : en l'occurrence, un niveau de langue populaire et relâché, qui, pour obéir à la loi du moindre effort, tend à bannir le complexe au profit du simple. Cette particularité langagière relève, d'après M. Banniard (2001 : 21) du «diasystème du latin parlé », et s'inscrit dans le «continuum latinophone » (Banniard $2012: 61)$.

Dans une optique d'évolution de la langue latine vers la langue française, c'est l'apparition progressive de traits rares et innovants dans un système de constructions usuelles, massivement majoritaires, qui mérite tout particulièrement de retenir notre attention. Ainsi, on constate l'apparition lente, à côté des propositions infinitives, de complétives assertives introduites par diverses conjonctions, à savoir quod, quia, ut et, plus rarement, quomodo, ainsi que l'affectation particulière de certains adverbes (de manière et de cause) ou particules à l'introduction des complétives interrogatives. En s'employant dans des contextes "critiques", qui admettent sans différence de sens appréciable deux lectures concurrentes, telle conjonction introductrice de subordonnée circonstancielle sera, par le processus de grammaticalisation, habilitée à introduire des complétives au sens du subordonnant universel "que » en français ou that en anglais ; tel subordonnant de sens hypothétique, déjà couramment affecté en latin tardif à l'introduction d'une interrogation indirecte totale, sera appelé à perdurer sous les deux formes «se » ou «si » comme introducteur exclusif du même type d'interrogative en français ou d'autres langues romanes ; enfin plusieurs thèmes en qu-, comme quare ou quomodo, passeront entre autres - par leur emploi en complétive interrogative de leur statut d'adverbe à celui de conjonction de subordination, voire de coordination ou d'élément grammatical d'une polyvalence foisonnante en roman. Ainsi, par un déplacement des «bornes » du champ de fluctuation (Banniard 2012: 73-74), des structures marquées en latinophonie moyenne deviennent non marquées en romanophonie, et cela, normalement, suite à une inversion des rapports de fréquence (structures minoritaires > structures majoritaires et vice-versa) d'un système à l'autre.

\section{Références bibliographiques}

BANNIARD M., 2001, «Le latin mérovingien. État de la question», in M. GOULLET, M. PARISSE (éds), Les historiens et le latin médiéval, Paris, Publications de la Sorbonne, p. 17-30.

\footnotetext{
${ }_{93}$ Pour plus de correspondances entre l'interrogatif de cause et la conjonction de coordination causale dans d'autres langues, voir J. B. Hofmann \& A. Szantyr $\left(1972^{2}: 542\right)$.

${ }^{94}$ Pour une étude préliminaire sur la même question avec un bilan faisant une distinction entre variations diachroniques, diatopiques et diaphasiques, voir C. Bodelot (à paraître b).
} 
BANNIARD M., 2012, « Le latin classique existe-t-il ? », in F. BiviLle, M. K. LhOMME, D. VALlat (éds), p. 57-78.

Biville F., LhommÉ M.-K., VALlat D. (éds), 2012, Latin vulgaire - latin tardif IX, Lyon, Maison de l'Orient et de la Méditerranée.

Bodelot C., 1987, L'interrogation indirecte en latin. Syntaxe - Valeur illocutoire - Formes, Paris, Société pour l'Information Grammaticale.

BODELOT C., 1990, Termes introducteurs et modes dans l'interrogation indirecte en latin de Plaute à Juvénal, «Bibliothèque de Vita Latina», Avignon, Aubanel.

BODELOT C., 1999, «L'indicatif dans l'interrogation indirecte en latin : auteurs anciens vs. auteurs tardifs », in H. PETERSMANN, R. KETTEMANN (éds), Latin vulgaire - latin tardif V, Heidelberg, C. Winter, p. 213-222.

Bodelot C., 2000, Espaces fonctionnels de la subordination complétive en latin. Étude morphosyntaxique et sémantico-énonciative, Louvain - Paris, Peeters.

Bodelot C., 2003, «L'interrogation indirecte », in C. BodELOT (éd.), p. 193-333.

Bodelot C., 2012, «Les emplois de quomodo chez Lucifer de Cagliari», in F. BiviLle, M.-K. LHOMMÉ, D. VALlAT (éds), p. 425-437.

BODELOT C., 2013, «La grammaticalisation de si en latin : de l'adverbe modal à la conjonction introduisant une subordonnée complétive ?», in C. BODELOT, H. GRUET-SKRABALOVA, F. TROUILLEUX (éds), p. 365-379.

Bodelot C., 2014, «Les propositions complétives dans la Chronique originale de Frédégaire (1. 4, chap. 1-90) », in P. Molinelli, P. CuZZOLIN, C. FEDRIANI (éds), p. 183-203.

Bodelot C., 2015, «Quōmŏdŏ : adverbe interrogatif et subordonnant, §7 : Descendance du lexème dans les langues romanes », in DHELL (Dictionnaire Historique et Encyclopédie Linguistique du Latin) [http://www.dhell.paris-sorbonne.fr/dictionnaire:quomodo7]

BODELOT C., à paraître a, "Particularités du discours indirect chez Frédégaire », in A. ORLANDINI, P. PocCetTI (éds), Actes de la Table ronde sur l'Oratio obliqua (17e Colloque International de Linguistique latine - Rome, 17-25 mai 2013).

BODELOT C., à paraître b, «Dico + proposition déclarative en hagiographie du IVe au XIIe s. ou de l'emploi toujours vivant de l'A.c.I. après un verbe de parole en médiolatin », in A. GARCIA LEAL, C. E. PRIETO ENTRIALGO (éds), Latin vulgaire - Latin tardif XI.

Bodelot C. (éd.), 2003, Grammaire fondamentale du latin. Tome X. Les propositions complétives en latin, Louvain - Paris - Dudley, MA, Peeters.

Bodelot C., Gruet-Skrabalova H., Trouilleux F. (éds), 2013, Morphologie, syntaxe et sémantique des subordonnants, Clermont-Ferrand, Presses Universitaires Blaise Pascal.

BolKestein A. M., 1989, "Parameters in the Expression of Embedded Predications in Latin », in G. Calboli (ed.), p. 3-35.

Calboli G., 1987, «Aspects du latin mérovingien », in J. HeRMAN (éd.), Latin vulgaire - Latin tardif: actes $d u 1^{e r}$ colloque international sur le latin vulgaire et tardif, Tübingen, Niemeyer, p. 19-35.

Calboli G. (ed.), 1989, Subordination and Other Topics in Latin. Proceedings of the Third Colloquium on Latin Linguistics, Amsterdam - Philadelphia, J. Benjamins.

Chomsky N., 1977, « On wh-movement», in P. W. Culicover, T. WAsow, A. Akmajian (eds.), Formal Syntax, New York, Academic Press.

CUZZOLIN P., 1994a, « On sentential complementation after verba affectuum », in J. HERMAN (ed.), Linguistic Studies on Latin. Selected Papers from the $6^{\text {th }}$ International Colloquium on Latin Linguistics, Amsterdam - Philadelphia, J. Benjamins, p. 201-210.

Cuzzolin P., 1994b, Sull'origine della costruzione 'dicere quod': aspetti sintattici e semantici, Firenze, « La Nuova Italia » Editrice.

DIEWALD G., 2002, «A model for relevant types of contexts in grammaticalization », in I. WISCHER, G. DiEwAld (eds.), New reflections on Grammaticalization, Amsterdam - Philadelphia, J. Benjamins, p. 103-121.

Dostie G., 2004, Pragmaticalisation et marqueurs discursifs. Analyse sémantique et traitement lexicographique, Bruxelles, De Boeck \& Duculot.

ERnout A., ThOMAS F. 1953², Syntaxe Latine, Paris, Klincksieck.

FIALON S, 2012, 'Mens immobilis'. Recherches sur le corpus latin des actes et des passions en Afrique romaine (IIe-VIe siécles). Thèse de doctorat, Univ. de Montpellier 3, 2012 (non publ.). 
FIALON S., à paraître, «Y a-t-il une Africitas ? Une analyse statistique et quantitative de trois passions de Maurétanie Césarienne», in S. Fialon, D. Longree, P. PIETQuin (éds), Langues anciennes et analyse statistique : cinquante ans après. Distances textuelles et intertextualités, Namur, Les Études classiques.

FLECK F., SZNAJDER L. (éds), 2015, Le discours rapporté en latin 1/2, De lingua latina, 10 (revue en ligne).

FRUYT M., 2015, «Le discours indirect en diachronie : l'évolution du réfléchi indirect en latin », in F. FLECK, L. SZNAJDER (éds).

GRECO P., 2008, «Accusatiuus cum infinitiuo and quod-clauses in the first and sixth books of the Historiae of Gregory of Tours, in R. WRIGHT (ed.), Latin vulgaire - latin tardif VIII, Hildesheim - Zürich - New York, Olms - Weidmann, p. 371-380.

GRECO P., 2012, La complementazione frasale nelle cronache latine dell'Italia centro-meridionale (secoli X-XII), Napoli, Liguori Editore.

GRECO P., 2014, «Sull'alternanza dei complementatori quod, quia e ut in dipendenza da verba dicendi et sentiendi in alcune agiografie di época merovingica (VI-VII secolo)», in P. Molinelli, P. CuZzolin, C. FedRiani (éds), p. 287-303.

GRECO P., 2015, « Le discours rapporté dans les actes notariés de la Langobardia minor (IXe siècle), in F. FLECK, L. SZNAJDER (éds).

HERMAN J., 1957, «Cur, quare, quomodo. Remarques sur l'évolution des particules d'interrogation en latin vulgaire », Acta Antiqua Academiae Scientiarum Hungaricae, 5, p. 369-377.

HERMAN J., 1963, La formation du système roman des conjonctions de subordination, Berlin, Akademie-Verlag.

HERMAN J., 1989, « Accusativus cum Infinitivo et subordonnée à quod, quia en latin tardif - nouvelles remarques sur un vieux problème », in G. CALBOLI (éd.), p. 133-152.

HOFMANN J. B., SZANTYR A., 1972², Lateinische Syntax und Stilistik, München, Beck.

KARLSEN E., 2001, The Accusativus cum infinitivo and Quod clauses in the Revelaciones of St. Bridget of Sweden, Bern et al., Peter Lang.

KÜHNER R., STEGMANN C., 1914², Ausführliche Grammatik der lateinischen Sprache, 2. Teil: Satzlehre, 1. und 2. Band, Hannover, Hahn (unveränd. reprograf. Nachdruck, Darmstadt, WBG, 1988).

MAYEN G., 1889, De particulis quod, quia, quoniam, quomodo, ut pro Acc. cum Infinitivo post verba sentiendi et declarandi positis, Dissertatio Inauguralis, Kiel.

Molinelli P, CuZzolin P., Fedriani C. (éds), 2014, Latin vulgaire - Latin tardif X, Bergamo, sestante edizioni.

Moralejo J. L., 1996, « Subjuntivo oblicuo y subordinación », in H. RoséN (ed.), Aspects of Latin, Innsbruck, Innsbrucker Beiträge zur Sprachwissenschaft, p. 287-296.

PHILIPPART DE FOY C., 2008, Hagiographie et statistique linguistique: étude d'un corpus de traductions médiolatines d'origine grecque, Thèse de doctorat, Univ. de Nice Sophia-Antipolis, 2008 (non publ.)

PHILIPPART DE FOY C., 2015, «Salsa au LASLA : lemmatisation et exploitation statistique de la Passio sanctae Salsae », in S. FIALON et J. MEYERS (éds.), La Passio sanctae Salsae (BHL 7467). Recherches sur une passion tardive d'Afrique du Nord, Bordeaux, Ausonius, p. 165-189.

PIRSON J., 1908, « Quomodo en latin vulgaire », in K. REUSCHEL , K. GRUBER (Hrsg.), Philologische und volkskundliche Arbeiten Karl Vollmöller zum 16. Oktober 1908, Erlangen, Junge, p. 61-74.

SERBAT G., 1981, «An a-t-il jamais un sens négatif ? », Vita Latina, 82, p. 2-5.

SERBAT G., 2003, « Les complétives en quod», in C. BODELOT (éd.), p. 528-753.

SORNICOLA R., 2014, «La costruzione DICO UT con valore evidenziale. Tra sviluppi stutturali interni al latino ed influenze esterne », in P. Molinelli, P. Cuzzolin, C. FEDRIANi (éds), p. 343-369.

STEPHENS L., 1985 [1986], «Indirect Questions in Old Latin: Syntactic and Pragmatic Factors Conditioning Modal Shift », Illinois Classical Studies, 10, 2, p. 195-214.

Stotz P., 1998, Handbuch zur lateinischen Sprache des Mittelalters. Vierter Band. Formenlehre, Syntax und Stilistik, München, Beck.

TOURATIER Ch., 1994, Syntaxe latine, Louvain-la-Neuve, Peeters.

VÄÄNÄNEN V., 198133, Introduction au latin vulgaire, Paris, Klincksieck.

VIELlaRD D., 2013, "'O $\tau$ l dans le Nouveau Testament, introducteur du discours direct: un subordonnant? », in C. BODELOT, H. GRUET-SKRABALOVA, F. TROUILLEUX (éds), p. 183-190. 
WANNER D., 1990, «Le subjonctif de subordination en latin vulgaire: questions indirectes et adverbiales temporelles, » in G. CALBOLI (éd.), Latin vulgaire - Latin tardif II, Tübingen, Niemeyer, p. 249-280.

Wielemans V., 2005, «L'évolution de comme et comment : le témoignage des grammaires et des dictionnaires de l'époque », clac 22, p. 1-34 (http://www.ucm.es/info/circulo/no22/wieleman.htm)

WIRTH-PÖLCHAU L., 1977, AcI und quod-Satz im lateinischen Sprachgebrauch mittelalterlicher und humanistischer Autoren, Inaugural-Dissertation, Friedrich-Alexander-Universität Erlangen Nürnberg.

Tableau en annexe :

Répartition des différents types de complétives dans le corpus hagiographique

(voir en PJ les deux tableaux Excel : «Textes rédigés en Italie » et «Textes rédigés en Gaule »; les insérer ici) 